\title{
Theory and Modeling of Planetary Dynamos
}

\author{
J. Wicht • A. Tilgner
}

Received: 8 June 2009 / Accepted: 15 February 2010 / Published online: 18 March 2010

(C) The Author(s) 2010

\begin{abstract}
Numerical dynamo models are increasingly successful in modeling many features of the geomagnetic field. Moreover, they have proven to be a useful tool for understanding how the observations connect to the dynamo mechanism. More recently, dynamo simulations have also ventured to explain the surprising diversity of planetary fields found in our solar system. Here, we describe the underlying model equations, concentrating on the Boussinesq approximations, briefly discuss the numerical methods, and give an overview of existing model variations. We explain how the solutions depend on the model parameters and introduce the primary dynamo regimes. Of particular interest is the dependence on the Ekman number which is many orders of magnitude too large in the models for numerical reasons. We show that a minor change in the solution seems to happen at $\mathrm{E}=3 \times 10^{-6}$ whose significance, however, needs to be explored in the future. We also review three topics that have been a focus of recent research: field reversal mechanisms, torsional oscillations, and the influence of Earth's thermal mantle structure on the dynamo. Finally we discuss the possibility of tidally or precession driven planetary dynamos.
\end{abstract}

Keywords Dynamo $\cdot$ Planets $\cdot$ Numerical model $\cdot$ Reversals

\section{Introduction}

Numerical dynamo simulations have flourished during the last 15 years. The work by Glatzmaier and Roberts (1995a) marked a first highlight after previous authors had proven the general validity of the concept (Zhang and Busse 1988). Since then, several new numerical codes have been developed that mostly aim at modeling the geodynamo. In a more

J. Wicht $(\bowtie)$

Max-Planck-Institut für Sonnensystemforschung, 37191 Kaltenburg-Lindau, Germany

e-mail:wicht@mps.mpg.de

A. Tilgner

Institut für Geophysik, Universität Göttingen, 37077 Göttingen, Germany 
recent development, dynamo simulations also attempt to explain the surprisingly different magnetic fields of the other planets in our solar system (Stanley and Bloxham 2004; Stanley et al. 2005; Takahashi and Matsushima 2006; Christensen 2006).

Numerical dynamos adequately model the strength and large scale geometry of several of the planetary fields in our solar system. Many details of the geomagnetic field as well as several aspects of its dynamics are also replicated successfully. This seems surprising since the numerical limitations force dynamo modelers to run their simulations at parameters that are far away from realistic values. For example, the fluid viscosity is generally many orders of magnitude too large in order to damp the small scale turbulent structures that can not be resolved numerically. There is therefore some doubt that dynamo simulations operate in the magnetostrophic regime thought to be characteristic for planetary dynamos. Recent scaling analysis, however, suggest that these doubts may no be warranted and that the success of the simulations is not coincidental (Christensen [doi:10.1007/s11214-009-9553-2], this issue).

Dynamo simulations have also proven to be a useful tool for understanding the magnetic fields and interior dynamics of planets. They are constantly refined to become more realistic and allow to explore an increasing number of phenomena on various length and time scales.

Several publications provide extensive overviews of the different aspects in numerical dynamo simulations (Braginsky and Roberts 1995; Jones 2000, 2007; Glatzmaier 2002) and discuss their success in modeling the geomagnetic field (Kono and Roberts 2002; Christensen and Wicht 2007). Here, we concentrate on more recent developments that mainly concern the geodynamo.

After a brief introduction into the fundamentals of the dynamo process in Sect. 2 we describe the mathematical formulation and the numerical methods employed for the simulations in Sect. 3. Section 4 and Sect. 5 provide an overview of solutions in non-magnetic convection and in the full dynamo problem, respectively. The following three chapters cover issues that were a focus of recent geodynamo simulations and highlight the power of today's numerical models. Section 6 discusses the dynamics of magnetic field reversals; a subject where numerical simulations are indispensable since paleomagnetic data provide very little insight. The implementation of the thermal boundary conditions imposed by Earth's lower mantle is an interesting attempt to make geodynamo simulations more realistic. We discuss the possible consequences in Sect. 8. Torsional oscillations are thought to form an important part of the decal geomagnetic field variations. They can also serve as a proxy for the magnetostrophic regime planetary dynamos are thought to operate in. Section 7 briefly reviews the topic and examines whether torsional oscillation can be found in dynamo simulations. In Sect. 9 we explore the possibility of tidally and precession driven dynamos. Section 10 closes the paper with a conclusion.

\section{Fundamentals}

Many review articles and textbooks summarize the aspects of magnetohydrodynamics and dynamo theory relevant for planetology, so that only a brief survey is appropriate here. A very readable introduction is provided by Davidson (2001). More mathematical treatments can be found in Moffat (1978) and Roberts (1987).

According to present day convictions, the induction equation of magnetohydrodynamics adequately represents magnetic field evolution in planetary interiors. This equation determines the behavior of magnetic field inside a liquid conductor in motion and derives from Maxwell's equations under the assumption that all motions are slow compared with the velocity of light and assuming Ohm's law is valid. The Hall effect for instance is excluded. 
One furthermore needs to assume that the material is characterized by a constant magnetic permeability. Thus no ferromagnetism is allowed. This chapter will deal with the simple case in which all magnetic material properties are negligible and the conductor has spatially uniform electrical conductivity $\sigma$. The induction equation for the magnetic field $\mathbf{B}(\mathbf{r}, t)$ then reads

$$
\frac{\partial}{\partial t} \mathbf{B}+\nabla \times(\mathbf{B} \times \mathbf{U})=\frac{1}{\mu \sigma} \nabla^{2} \mathbf{B}
$$

where $\mu$ is the magnetic permeability of vacuum and $\mathbf{U}(\mathbf{r}, t)$ is the velocity of the conductor. To pose a mathematically meaningful problem, boundary conditions must also be specified. Vacuum boundary conditions are frequently used, which is appropriate if one models the mantle surrounding the core as a perfect insulator.

The induction equation is more useful in dimensionless form. If one expresses all lengths in multiples of a length $L$ characterizing the size of the volume filled with liquid conductor, and all times in multiples of $L / \mathrm{u}$ where $\mathrm{u}$ is the characteristic velocity of the conductor, one arrives at

$$
\frac{\partial}{\partial t} \mathbf{B}+\nabla \times(\mathbf{B} \times \mathbf{U})=\frac{1}{\mathrm{Rm}} \nabla^{2} \mathbf{B}
$$

in which all quantities (length, time, velocity, magnetic field) are dimensionless. The magnetic Reynolds number

$$
\mathrm{Rm}=\mu \sigma L \mathrm{u}
$$

is a rough measure for the ratio of magnetic induction to Ohmic dissipation.

The induction equation can be solved as an independent kinematic problem or as part of a full self consistent approach. In the kinematic problem, $\mathbf{U}$ is prescribed, so that the dynamo equation (2) becomes an eigenproblem. One can then explore at which critical magnetic Reynolds number the magnetic field starts to grow.

In the full problem that we will discuss in the following section, additional equations such as the Navier-Stokes equation have to be used in order to compute the velocity field $\mathbf{U}$ from a prescribed driving mechanism, as for example thermal convection. The velocity field is affected via the Lorentz force by the magnetic field it generates. This back reaction will limit the exponential field growth found in the kinematic problem. The self-consistent approach is a very intricate problem which can only be solved numerically in most cases of interest. The kinematic dynamo problem is much simpler in that it does not prescribe the driving mechanism but the velocity field itself, which is considered as a given. This approach is useful if one wants to find out whether a certain motion is capable of dynamo action at all.

Thanks to its simplicity, kinematic theory gave rise to several useful notions. There are a few mathematically rigorous statements, known as antidynamo theorems, about situations in which no dynamo action can occur. For example, it is known that a purely axisymmetric field cannot result from dynamo action. In terms of positive results, there are two well established scenarios for field amplification. The first scenario relies on the Alfvén theorem which states that in a perfect conductor, magnetic field lines are frozen into the fluid. Two fluid particles lying on a magnetic field line will stay connected by a magnetic field line forever. Stretching of field lines locally increases the magnetic energy density. Strongest field amplification thus occurs in flows with Lagrangian chaos, in which two initially nearby fluid parcels separate exponentially in the course of time. This mechanism obviously only operates at high magnetic Reynolds numbers. 
The second scenario is in principle not restricted to any particular range of $\mathrm{Rm}$, but its theory is best developed for small $\mathrm{Rm}$. The so called $\alpha$-effect relates the magnetic field and the induction term (the second term in the induction equation above) at large scales. This formalism circumvents the need to compute the magnetic field at small scales. The effects of small scales are subsumed in the $\alpha$-effect, which in turn is expressed mathematically in the form of the $\alpha$-tensor. The entries in this tensor can in most circumstances only be chosen heuristically. But there is one well studied example of a 2D periodic flow first investigated by G.O. Roberts (1972) for which the $\alpha$-tensor can be computed asymptotically for small $\mathrm{Rm}$. This flow is helical, and one can give a handwaving description of the amplification mechanism in terms of magnetic flux tubes twisted by helical vortices. Since rotating flows always contain helicity, and because the magnetic Reynolds numbers in planetary dynamos tend to be moderate, most interpretations of these dynamos (or their numerical models) use the concept of an $\alpha$-effect in a helical flow. On the other hand, it should be pointed out that helicity is not a prerequisite for dynamo action, even in the restricted class of $2 \mathrm{D}$ periodic dynamos. However, non-helical 2D periodic dynamos need a higher Rm than comparable helical dynamos (Tilgner 2004).

\section{Mathematical Model}

\subsection{Basic Equations}

Self-consistent dynamo models simultaneously solve for the convective fluid flow and magnetic field generation in a rotating spherical shell (see Fig. 1). The shell is confined by an outer boundary at radius $r_{o}$ which represent the core-mantle boundary in terrestrial planets or the transition to an electrically insulating outer envelope in the gas planets. An inner boundary at $r=r_{i}$ models the surface of the solid iron cores of terrestrial planets or the rocky cores in the gas giants. The problem is solved in a reference frame corotating with the planetary rotation about the $z$-axis. The convective motions are driven by density differences due to variations in chemical composition and in temperature. Compositional convection arises in terrestrial planets that have a growing inner core. The lighter constituents mixed into the iron/nickel core alloy have a smaller solubility in the solid than in the liquid phase. They are therefore rejected at the inner-core freezing front and give rise to compositional density differences. The latent heat associated with the phase change provides an important source

Fig. 1 Geometry of a typical dynamo model for a terrestrial planet with a solid inner core

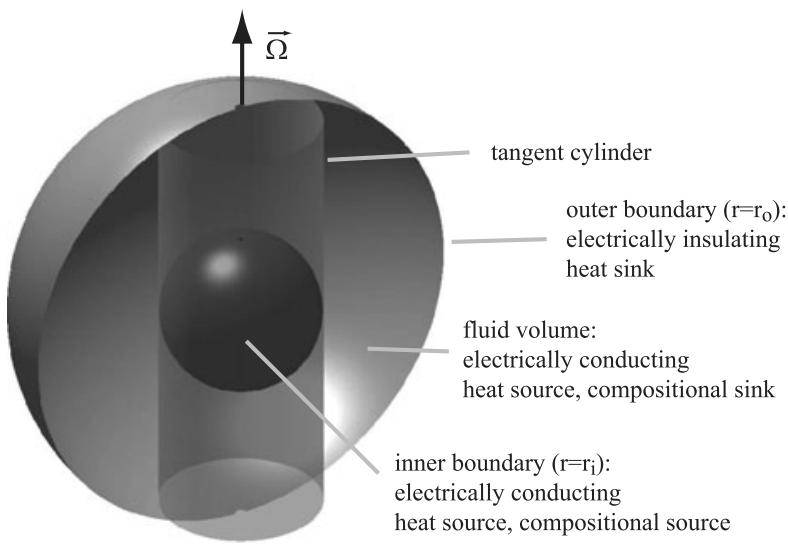


for thermal convection. Other potential sources are secular cooling and the heat production from radiogenic elements. Only the temperature variations that exceed the adiabatic gradient have to be considered in the models since only these give rise of convection.

Dynamo codes solve for the disturbances around a reference state which is assumed to be hydrostatic, well mixed, and has no magnetic field. The statement 'well mixed' implies that the convection is strong enough to practically homogenize the chemical composition and entropy. The reference state is thus adiabatic. This approach is justified since the disturbances are indeed very small. The convective temperature disturbances, for example, amount to only $10^{-6}$ times the adiabatic temperature drop across Earth's outer core (Braginsky and Roberts 1995; Jones 2007).

The planets secular cooling and differentiation not only provide the necessary heat and compositional fluxes to drive the dynamo but will also cause the references state to evolve. This evolution is so slow that it can be neglected on the time scales typical dynamo models are concerned with (Labrosse 2003; Lister and Buffett 1995).

Developing the general set of equation for the dynamo problem is beyond the scope of this paper; we refer to Braginsky and Roberts (1995). Here, we restrict ourselves to the simplified system provided by the Boussinesq approximation where the dissipation number,

$$
\mathrm{Di}=-\frac{L}{\bar{T}} \frac{\partial \bar{T}}{\partial r},
$$

and the compressibility parameter,

$$
\mathrm{Co}=-\frac{L}{\bar{\rho}} \frac{\partial \bar{\rho}}{\partial r}
$$

are assumed to vanish. The Boussinesq approximation thus neglects the temperature and density variations in the reference state. Here, $L=r_{o}-r_{i}$ is the depth of the shell and bars over the variables refer to the reference state. Di and Co are identical to the inverse reference temperature and density scale heights, two alternative parameters sometimes used in dynamo theory.

In Earth's outer core, the density increases by about $25 \%$ from top to bottom which translates to a compressibility parameter of $\mathrm{Co} \approx 0.2$. The relative temperature increase is of comparable magnitude and yields $\mathrm{Di} \approx 0.3$ (Anufriev et al. 2005). Similar values can be expected for the dynamo regions of the other terrestrial planets. While both these nondimensional numbers are not very small, they nevertheless indicate that the Boussinesq approximation may yield a fair approach. Since it considerably simplifies the problem, the Boussinesq approximation has been employed by the great majority of numerical dynamo simulations, even for gas planets where the applicability is questionable.

Neglecting the temperature and density variation in the reference state not only implies that $\bar{\rho}$ is homogeneous and constant, the same also holds for the other physical properties: the electrical conductivity $\sigma$, the thermal expansivity $\alpha$, the dynamic viscosity $\eta$, the thermal conductivity $k$, the magnetic permeability $\mu$, and the heat capacity $c_{p}$. The Boussinesq approximation moreover has the consequence that viscous heating and Joule heating are neglected and that only the density variations due to temperature and compositional differences in the buoyancy force are retained.

In the following, we describe only one possible non-dimensional form for the set of equations which largely follows Christensen and Aubert (2006). The readers should be aware that there are several different ways of choosing the scaling factors and the non-dimensional parameters, which often leads to confusion when comparing different models. We use $L$ as the 
length scale, $\Omega^{-1}$ as the time scale, the codensity jump $\Delta c$ across the shell as the codensity scale, and $(\bar{\rho} \mu)^{1 / 2} \Omega L$ as the magnetic scale.

The mathematical dynamo model in the Boussinesq approximation then comprises the Navier-Stokes equation

$$
\frac{d \mathbf{U}}{d t}=-\nabla P-2 \hat{\mathbf{z}} \times \mathbf{U}+\mathrm{Ra}^{\star} \frac{r}{r_{o}} C \hat{\mathbf{r}}+(\nabla \times \mathbf{B}) \times \mathbf{B}+\mathrm{E} \nabla^{2} \mathbf{U},
$$

the induction equation

$$
\frac{\partial \mathbf{B}}{\partial t}=\nabla \times(\mathbf{U} \times \mathbf{B})+\frac{\mathrm{E}}{\mathrm{Pm}} \nabla^{2} \mathbf{B},
$$

the codensity equation

$$
\frac{d C}{d t}=\frac{\mathrm{E}}{\operatorname{Pr}} \nabla^{2} C+q
$$

the continuity equation

$$
\nabla \cdot \mathbf{U}=0
$$

and the magnetic continuity equation

$$
\nabla \cdot \mathbf{B}=0
$$

Here $d / d t$ stands for the substantial time derivative $\partial / \partial t+\mathbf{U} \cdot \nabla$.

The model is controlled by five dimensionless numbers: Ekman number

$$
\mathrm{E}=\frac{v}{\Omega L^{2}},
$$

modified Rayleigh number

$$
\mathrm{Ra}^{\star}=\frac{\bar{g}_{o} \Delta c}{\Omega^{2} L}
$$

Prandtl number

$$
\operatorname{Pr}=\frac{v}{\kappa}
$$

magnetic Prandtl number

$$
\operatorname{Pm}=\frac{v}{\lambda}
$$

and aspect ratio

$$
a=r_{i} / r_{o} .
$$

We have introduced three diffusivities here: the magnetic diffusivity $\lambda=1 / \sigma \mu$, the viscous diffusivity $v=\eta / \bar{\rho}$, and the thermal diffusivity $\kappa=k /\left(c_{p} \bar{\rho}\right) \cdot \bar{g}_{o}$ is the references gravity at the outer boundary. $\mathrm{Ra}^{\star}$ is connected with the more classical Rayleigh number

$$
\mathrm{Ra}=\frac{\bar{g}_{o} \Delta c L^{3}}{\kappa v}
$$

via $\mathrm{Ra}^{\star}=\mathrm{Ra}^{2} \operatorname{Pr}^{-1}$. 
Above, we have used an additional simplification that has been adopted in several dynamo models of terrestrial planets: The density variations due to temperature and composition are combined into one variable, the codensity

$$
c=\alpha T+\gamma \chi .
$$

Here, $\chi$ describes the compositional disturbances around the reference state compositions $\bar{\chi}=m_{L} /\left(m_{L}+m_{H}\right)$. We assume a simplified binary model composition with heavy constituents (iron, nickel) of total mass $m_{H}$ and light constituents (sulfur, oxygen, carbon) of total mass $m_{L}$. The compositional expansivity is given by $\gamma=-\bar{\rho}\left(\rho_{H}-\rho_{L}\right) / \rho_{H} \rho_{L}$, where $\rho_{H}$ and $\rho_{L}$ are the densities of heavy and light elements in the liquid core, respectively. Note that $T$ in (17) refers to the dimensional temperature and that the dimensionless codensity in (8) is given by $C=c / \Delta c$.

Describing the evolution of temperature and composition by the combined equation (8) assumes that both quantities have similar diffusivities. This seems like a daunting simplification since the chemical diffusivity may be three orders of magnitude smaller than the thermal one (Braginsky and Roberts 1995). The approach is often justified with the argument that the small scale turbulent mixing, which can not be resolved by the numerical codes, may result in larger effective turbulent diffusivities of comparable magnitude (Braginsky and Roberts 1995). Consequently, the 'turbulent' Prandtl numbers would then be of order one. Some studies suggest that the differences in diffusivities may have interesting implications (Busse 2002) and the potential effects on planetary dynamo simulations remains to be explored.

In Jupiter, the pressure increases by over a factor of 200 over the convective gas envelopes which we consider to start at 1000 bar (Evonuk 2008). This demonstrates that the Boussinesq approximation does not apply in gas planets. The implementation of the density variations in a dynamo model and the possible effects that go along with such a strong density stratification are, for example, discussed in Braginsky and Roberts (1995), in Anufriev et al. (2005), and in (Stanley and Glatzmaier [doi:10.1007/s11214-009-9573-y], this issue).

\subsection{Boundary Conditions and Driving Modes}

The differential equations comprising the mathematical dynamo problem can only be solved when supplemented with an adequate set of boundary conditions. For the flow $\mathbf{U}$, either rigid or free slip conditions are used. In both cases the radial flow component is forced to vanish and the differences lie in the horizontal components. They must match the motion of the boundary for rigid conditions, for example the solid body rotation of an inner iron core around the planetary rotation axis. For free slip conditions the horizontal components of the viscous stress are force to vanish which allows for a discontinuity in the horizontal flow itself. Rigid flow conditions are most appropriate for the dynamo regions of terrestrial planets and the boundary to a rocky core in gas planets.

In Jupiter and Saturn, the outer boundary of the dynamo region is marked by the hydrogen transition from the electrically insulating to the conducting state. In Uranus and Neptune, ionically conducting liquid ice layers seem the most likely candidates for the dynamo regions (Stanley and Bloxham 2006). Lorentz forces and higher densities are thought to slow down the dynamics in the conducting layers. Since the insulating layer can thus more easily adapt to the conducting layer, stress free outer boundary conditions seem appropriate. However, the dynamical separation of the conducting and insulating envelopes in gas planets is little understood. For example in Saturn and Jupiter, the conductivity decreases only gradually with pressure (Liu et al. 2008) which questions the strict separation of the two layers. 
Some authors (Kuang and Bloxham 1997; Busse and Simitev 2005) prefer to employ free slip conditions when modeling the geodynamo in order to exclude the too thick boundary layers in the numerical models. We come back to this point in Sect. 5.3.

In terrestrial planets, the conductivity of the mantle is orders of magnitudes lower than that of the core. Like in the gas planets, the magnetic field must therefore match a potential field at the outer boundary. The same applies at the boundary to an electrically insulating inner core. For modeling the magnetic field in conducting solid inner cores a simplified induction equation (7) has to be solved that includes diffusive effects and the advection due to the inner core rotation (Christensen and Wicht 2007). Inner core and outer core magnetic fields have to match at the interface.

Most dynamo models employ the simplifying codensity description and use either fixed flux or fixed codensity conditions. This is done for simplicity and has no physical basis. Kutzner and Christensen (2000) suggest that the solutions are not very sensitive to the boundary conditions as long as the inner core boundary is the main codensity source. Their examination was restricted to laterally homogeneous conditions in a few cases and more work is needed to clarify the influence of thermal and compositional boundary conditions in the future. We outline the proper conditions for gas and terrestrial planets in the following, assuming that two separate equations of the form (8) may be used to describe the evolution of temperature $T$ and composition $\chi$, respectively.

Fixed temperature conditions seem appropriate for the outer boundary of gas planets, provided the dynamics of the insulating layer is vigorous enough to homogenize the temperature. At the inner boundary, a fixed heat flux $F_{i}^{T}=f_{i} k \partial T / \partial r$ should model secular cooling and potential radioactive heating of the rocky core. Here, $f_{i}$ is the inner core surface, and $\partial T / \partial r$ refers to the spherical symmetric contribution, the other contributions being zero.

In terrestrial planets, the appropriate boundary conditions for temperature and composition are more complex. The inner core is a source for heat and compositional codensity represented by the respective fluxes (see Fig. 1). Since the chemical core elements cannot penetrate the mantle the composition flux has to vanish here: $F_{o}^{\chi}=0$. The outer thermal boundary condition is imposed by the slowly evolving mantle which changes on time scales of tens to hundreds of million years. The much faster changing core is virtually isothermal on these time scales so that horizontal differences in the lower mantle temperature translate into a cmb heat flux amplitude and pattern. The resulting laterally inhomogeneous temperature boundary layer at the base of the mantle is sometimes identified with the $\mathrm{D}^{\prime \prime}$ layer. We discuss the possible consequences for the dynamo process in Sect. 8.

The thermal and compositional boundary conditions at the interface to a growing inner iron core are more involved. The compositional flux $F_{i}^{\chi}$ and heat flux $F_{i}^{T}$ released from the inner core boundary are proportional to the inner core growth rate. The growth rate in turn depends on the local cooling rate $\bar{\rho} c_{p} d T / d t$ and compositional rate of change $d \chi / d t$, i.e. it depends on how fast the outer core convection removes heat and light elements (Braginsky and Roberts 1995). These relations yield two equations that connect temperature flux and compositional flux to $d T / d t$ and $d \chi / d t$ and form the appropriate boundary conditions (Braginsky and Roberts 1995; Glatzmaier and Roberts 1996a; Jones and Roberts 2000). They allow for lateral variations in the flux from the inner core that translate to differences in the inner core growth rate. Such variations are also permitted in codensity formulations that impose a fixed codensity at the inner boundary. Aubert et al. (2008a) use such a dynamo model to show that the variations may explain hemispherical differences in the inner-core seismic signal, as we will discuss in Sect. 8. 
The homogeneous volumetric source/sink term $q$ in (8) determines the ratio of volumetric driving to bottom driving and, strictly speaking, is an additional system parameter. It serves to model volumetric sources like secular cooling and radioactive heating and has the additional task to prevent an evolution of the reference state by balancing the compositional flux $F_{i}^{\chi}$ with a sink term (see Fig. 1). The most commonly used setup employs $q=0$ and thus models a purely thermal heat flux from the inner core boundary. Consequently, the codensity (17) could be replaced by $\alpha T$ in the mathematical model which yields a form of equation more typically found in the dynamo literature. Choosing $q \neq 0$ will add volumentric sources which increase the outer boundary heat flux. When inner and outer boundary flux conditions are used they have to fulfill $F_{o}=-F_{i}+V q$, where $V$ is the volume of the shell. For fixed codensity conditions, the inner boundary flux $F_{i}$ and/or the outer boundary flux $F_{o}$ are controlled by the vigor of convection, i.e. by the Rayleigh number and the other system parameters. Kutzner and Christensen (2002) model pure chemical convection by imposing a vanishing flux $F_{o}=0$ at $r_{o}$, by choosing $q<0$, and by using a fixed codensity condition at $r_{i}$. For gas planets and terrestrial planets without a growing inner core $q>0$ models the homogeneous secular cooling or a homogeneous radiogenic heating as the only driving forces. Whether radiogenic heating is sizable or can be neglected is still a matter of debate (Labrosse [doi:10.1007/s11214-010-9630-6], this issue). Secular cooling can be modeled by a uniform decrease of an adiabatic reference temperature throughout the core (Labrosse 2003; Nimmo 2007). The errors made by approximating the inner core temperatures by an adiabat are very small. The associated inner core boundary condition is a fixed heat flux $F_{i}=V_{i} q$, where $V_{i}$ is the inner core volume. Note that we have neglected a potential difference of the radiogenic heat source densities in inner and outer core.

A deviation from fixed codensity conditions requires a different codensity scale and thus a different Rayleigh number than (12) (Kutzner and Christensen 2002; Aubert et al. 2008a). For example, when imposing a dimensional flux density $f$ at either boundary a possible codensity scale is $L f / \kappa$ which yields the Rayleigh number:

$$
\mathrm{Ra}^{\prime}=\frac{\bar{g}_{o} f}{\Omega^{2} \kappa}
$$

Further discussions of the different driving sources and the respective boundary conditions in terrestrial planets can be found elsewhere (Lister and Buffett 1995; Wicht et al. 2007) (Labrosse [doi:10.1007/s11214-010-9630-6], this issue).

\subsection{Parameters, Force Balance, and Scaling}

Table 1 compares the non-dimensional parameters for Earth with those for some typical dynamo simulations that we will explore in more detail in the following sections. Obviously, dynamo simulations operate at Ekman numbers and magnetic Prandtl numbers which are orders of magnitude too large. The reason is, that the turbulent small scale flow thought to be present in planetary dynamo regions can not be resolve with today's computers. The larger Ekman numbers used in the simulations reflect an increase in viscosity which helps to damp away the smaller scale flows.

The obvious differences in parameters complicate a direct comparison of the simulation results with the planetary magnetic fields. The numerical models are therefore typically discusses in term of dimensionless numbers that quantify important dynamo properties. Like the dimensionless parameters introduced in Sect. 3, these have the advantage of combining different physical into quantities that better characterize the dynamics. The Ekman number 
Table 1 List of parameters and properties for some of the dynamo simulations presented here. Reynolds number $\mathrm{Rm}$ Elsasser number $\Lambda$, and local Rossby number $\operatorname{Ro}_{\ell}$ are based on time average rms energies in the dynamo region. The relative dipole strength $\mathrm{D}$ at the outer boundary is also a time averaged value. Earth values are takes from (Jones 2007), the $\mathrm{Ro}_{\ell}$ value is suggested by Christensen and Aubert (2006). Earth's Rayleigh number is hard to constrain but thought to be very much supercritical (Gubbins 2001)

\begin{tabular}{lllllllllll}
\hline Name & E & Ra & Pm & Pr & Ro & Rm & $\Lambda$ & Ro $\ell$ & D & reversing? \\
\hline E3a & $10^{-3}$ & $1.10 \times 10^{-1}$ & 5 & 1 & $9 \times 10^{-3}$ & 46 & 9 & $3.4 \times 10^{-2}$ & 0.8 & no \\
E3b & $10^{-3}$ & $4.50 \times 10^{-1}$ & 10 & 1 & $4 \times 10^{-2}$ & 392 & 25 & $1.1 \times 10^{-1}$ & 0.3 & yes \\
E5b & $3 \times 10^{-5}$ & $1.08 \times 10^{-1}$ & 1 & 1 & $10^{-2}$ & 405 & 8 & $9.7 \times 10^{-2}$ & 0.6 & no \\
E5c & $3 \times 10^{-5}$ & $1.35 \times 10^{-1}$ & 1 & 1 & $2 \times 10^{-2}$ & 607 & 4 & $1.4 \times 10^{-1}$ & 0.09 & yes \\
E5F & $3 \times 10^{-5}$ & $5.04 \times 10^{-2}$ & 1 & 1 & $8 \times 10^{-3}$ & 260 & 4 & $6.5 \times 10^{-2}$ & 0.8 & no \\
E6 & $3 \times 10^{-6}$ & $9.00 \times 10^{-3}$ & 0.5 & 1 & $2 \times 10^{-3}$ & 261 & 3 & $2.3 \times 10^{-2}$ & 0.7 & no \\
Earth & $10^{-15}$ & & $3 \times 10^{-7}$ & 1 & $2 \times 10^{-6}$ & 500 & 1 & 0.09 & $0.3-0.6$ & yes \\
\hline
\end{tabular}

can be interpreted as a measure for the relative importance of viscous forces compared to the Coriolis force. Two additional non-dimensional numbers quantify the relative importance of inertial effects and magnetic forces in the Navies-Stokes equation (6), once again in comparison to the Coriolis force. These are the Rossby number

$$
\mathrm{Ro}=\frac{\mathrm{u}}{L \Omega}
$$

and the Elsasser number

$$
\Lambda=\frac{b^{2}}{\bar{\rho} \Omega \mu \lambda} .
$$

$\mathrm{u}$ and $\mathrm{b}$ refer to typical dimensional flow and magnetic field amplitudes. Note that the Rossby number Ro is identical to the non-dimensional flow amplitude in the scaling chosen here. The magnetic Reynolds number $\mathrm{Rm}=\mathrm{u} L / \lambda$ has already been introduced in Sect. 2 as an important measure for the ratio of magnetic induction to diffusion.

Planetary dynamos are thought to operate in the magnetostrophic regime. This refers to a specific first order force balance in the Navier-Stokes equation between Coriolis force, pressure gradient, Lorentz force, and buoyancy. Viscous forces and inertial forces are deemed negligible in comparison because the Ekman numbers and Rossby numbers of planetary dynamo regions are small. An Elsasser number of order one testifies that the Lorentz force contributes to the first order balance. The magnetostrophic balance can be understood as an extension of the geostrophic balance in non-magnetic systems where Coriolis force and pressure gradient constitute the main force balance with buoyancy contributing to the radial accelerations.

Elsasser number, Ekman number, and Rossby number can only provide rough estimates of the true force balances and may actually fail in many instances. The Rossby number seems to do a particularly bad job in estimating the importance of the nonlinear advection $\mathbf{U} \cdot \nabla \mathbf{U}$ in the Navier-Stokes equation (6). This non-linearity can give rise to so-called Reynolds stresses that transport energy from smaller to larger scales or vice versa. Reynolds stresses are the result of a statistically persistent correlation between non-axisymmetric flow components. Very prominent examples for their potential power are the fierce zonal winds observed on Jupiter and Saturn (Christensen 2002; Heimpel et al. 2005). The length scale dependence, introduced by the $\nabla$ operator, can thus 
not be neglected when estimating the importance of non-linear advection. Christensen and Aubert (2006) therefore suggest to use the local Rossby number

$$
\operatorname{Ro}_{\ell}=\frac{\mathrm{u}}{\Omega \ell},
$$

instead of Ro. The length scale $\ell$ represents a weighted average base on the spherical harmonic decomposition of the flow:

$$
\ell=r_{o} \pi /\langle n\rangle
$$

with

$$
\langle n\rangle=\frac{\sum_{n} n E_{k}(n)}{\sum_{n} E_{k}(n)},
$$

where $E_{k}(n)$ is the kinetic energy carried by all modes with spherical harmonic degree $n$.

Local Rossby number $\mathrm{Ro}_{\ell}$, Elsasser number $\Lambda$, and magnetic Reynolds number $\mathrm{Rm}$ are not input parameters but measure properties of the solution. Rather than stating absolute values of magnetic field strength or flow speed dynamo model results are typically discussed in terms of Elsasser number $\Lambda$ and magnetic Reynolds number Rm. Realistic values of $\Lambda$ and $\mathrm{Rm}$ are thought synonymous with realistic values of $\mathrm{b}$ and $\mathrm{u}$. Concerning the magnetic field strength this implies that $\mathrm{b}$ depends on $\Omega, \bar{\rho}, \mu$, and $\lambda$ since $\mathrm{b}=(\Lambda \Omega \bar{\rho} \mu \lambda)^{1 / 2}$. Some authors rescale the dimensionless field strength in their solutions by assuming realistic values for all these four properties (Wicht 2005; Christensen 2006; Wicht et al. 2009). The length scale is provided via a realistic shell thickness $L$, and the magnetic diffusion time $t_{\lambda}=L^{2} / \lambda$ typically serves to rescale time. However, Ekman number and magnetic Prandtl number of a numerical model already fix the ratio between the rotation period and $t_{\lambda}$ to $\mathrm{E} / \mathrm{Pm}=\lambda / \Omega L^{2}$ which is typically many orders of magnitude too large. The described field strength scaling therefore amounts to an extrapolation of E/Pm, sometimes called the magnetic Ekman number $\mathrm{E}_{\lambda}$, to the much smaller planetary values (Glatzmaier 2002). Realistic flow amplitudes are assumed for realistic magnetic Reynolds numbers when $t_{\lambda}$ serves to rescale time.

Christensen and Aubert (2006) realized that the Elsasser number in their numerical simulations is not necessarily of order one but varies over three orders of magnitude. The extrapolation for many of their model would therefore yield unrealistic field strengths despite that fact that they have very similar characteristics otherwise. Wicht and Christensen (2010) argue that the Elsasser number may not always be a good proxy for the force balance in the dynamo region and demonstrate that the numerical models may still obey the magnetostrophic force balance for Elsasser numbers significantly larger than one. Motivated by their findings, Christensen and Aubert (2006) proceed to develop a scaling where the magnetic field and flow amplitudes depend on the heat flux out of the dynamo region (Christensen and Aubert 2006). This scaling seems to do a better job in subsuming the simulation results and correctly predicts the field strength for several planetary dynamos and even for the dynamos of fast rotating stars (Christensen et al. 2009). We refer to (Christensen [doi:10.1007/s11214-009-9553-2], this issue) for a review of the various scaling laws that have been suggested over the years.

\subsection{Model Variations}

The most commonly adopted dynamo modeling approach, which we will refer to as the 'standard model' in the following, solves the full set of equations in the Boussinesq approach, has an Earth like inner core size, employs a codensity description, and uses rigid 
flow and fixed codensity boundary conditions. The inner core has either been treated as an electrical insulator or a conductor and Christensen et al. (2001) defines a benchmark for both cases. Minor variations concern the use of different flow and codensity boundary conditions. Some more involved variations characterize several other types of convection driven dynamo models.

The Kuang and Bloxham model (Kuang and Bloxham 1997, 1999) adopts the Boussinesq approximation, uses stress free boundary conditions, and neglects the non-axisymmetric inertial terms in the Navier-Stokes equation in order to possibly better approach the magnetostrophic force balance. The axisymmetric inertial terms are retained to allow for the torsional oscillations discussed in Sect. 7. Another feature of this model is the use of hyperdiffusivity, a numerical trick that increases the diffusivity with spherical harmonic degree $l$ to stronger damp small scale contributions. The Kuang \& Bloxham model has been adopted for several publications since (Kuang 1999; Stanley and Bloxham 2004, 2006; Stanley et al. 2005, 2007).

An early Glatzmaier and Roberts model neglects all inertial terms in a Boussinesq approach (Glatzmaier and Roberts 1995a, 1995b) and also employs hyperdiffusion. The later Glatzmaier and Roberts model retains compressible effects in an anelastic approximation, allows for axisymmetric inertia and also uses hyperdiffusivity. It furthermore comprises several advanced features like separate equations for describing the evolution of temperature and composition and a model of inner-core growth along with the appropriate temperature and composition boundary conditions (3.2). The influence of these advanced features has not been properly explored so far. This later Glatzmaier and Roberts model has been employed in several studies during the last years (Glatzmaier and Roberts 1996a, 1996b; Glatzmaier et al. 1999; Coe et al. 2000; Roberts and Glatzmaier 2001; Ogden et al. 2006).

The Busse group models employ the Boussinesq approach, typically use stress free boundary conditions, and are driven a mixture of volumetric and bottom buoyancy sources (Ardes et al. 1996; Wicht and Busse 1997; Grote et al. 1999, 2000; Grote and Busse 2000; Simitev and Busse 2005; Busse and Simitev 2006, 2008). The Kageyama type model describes the dynamo medium as an ideal gas and retains weak compressibility effects (Kageyama and Sato 1995, 1997; Kageyama et al. 1999, 2008; Ochi et al. 1999; Li et al. 2002; Kageyama and Yoshida 2005; Nishikawa and Kusano 2008).

\subsection{Numerical Methods}

The most common method for solving the numerical dynamo problem is a pseudo-spectral approach. More recently, several authors have adopted so-called local methods which promise to be better adapted to massive parallel computing.

In the pseudo-spectral implementations, all variables are defined on a numerical grid and also have a global spectral representation. Spherical harmonic functions $Y_{l m}(\theta, \phi)$ are the obvious choice for the spectral representation in colatitude $\theta$ and longitude $\phi$ where $l$ and $m$ denote degree and order. Chebychev polynomials $C_{n}(r)$ are typically chosen for the spectral representation in radius. When applied appropriately, they provide a denser radial grid near the inner and outer boundaries where boundary layers may have to be sampled (Glatzmaier 1984; Christensen and Wicht 2007).

The equation system is time-stepped in spherical harmonic and radial space $(l, m, r)$ using a mixed implicit/explicit scheme where non-linear terms as well as the Coriolis force are treated explicitely in an Adams-Bashforth time step. This guarantees that all spherical harmonic modes $(l, m)$ decouple. A Crank-Nicolson implicit scheme completes the 
time integration for the remaining contributions. The nonlinear terms are evaluated on the local grid space, which requires a transform from the $(l, m, r)$ to the $(\theta, \phi, r)$ representation and back. Fast Fourier transforms can be applied for the longitudinal dependence. The Gauss-Legendre transformation employed in the latitudinal direction are significantly more time consuming and can considerably slow down the computation for highly resolved cases.

All partial derivatives are evaluated in spectral space which guarantees a high degree of exactness. Local approaches use finite difference or finite element methods whose lower precision can require considerably denser grids (Christensen et al. 2001). Another advantage of the pseudo-spectral codes is the straightforward implementation of the magnetic boundary conditions at the outer boundary. Local methods either use the artificial condition that the horizontal field has to vanish at the interface or have to rely on additional numerical procedures (Matsui and Okuda 2005; Wicht et al. 2009).

Pseudo spectral methods use a poloidal/toroidal decomposition of flow and magnetic field. For example, the flow decomposition reads

$$
\mathbf{U}=\nabla \times \hat{\mathbf{r}} w+\nabla \times \nabla \hat{\mathbf{r}} v
$$

where $w$ and $v$ are the toroidal and poloidal flow potentials, respectively. The continuity equations (9) and (10) are then fulfilled automatically. Local approaches, however, typically use primitive variables, i.e. the individual flow and magnetic field components, in order to avoid the additional derivatives introduced by (24). Special numerical measures are then required to assure that the continuity equations are fulfilled (Harder and Hansen 2005). Local methods also face the problem that the grid points in the natural longitude/latitude grid come very close near the poles which may cause numerical instabilities. These instabilities can either be damped (Kageyama and Sato 1995, 1997) or other grids have to be used that provide a more even coverage (Kageyama and Yoshida 2005; Harder and Hansen 2005).

The disadvantage of the pseudo-spectral approaches is the large communication overhead between the individual processors in a parallel computer. Each processor needs the full solution information in every time step. In the local methods a processor that represents a numerical grid point or volume element only requires the information from processors representing neighboring grid points. The local methods have not yet proven to be faster than pseudo-spectral methods in the dynamo context, but this may change on massively parallel computing systems with several thousand processors. A recent overview of local methods can be found in Wicht et al. (2009). More information on the pseudo-spectral method can be found in Glatzmaier (1984) and Christensen and Wicht (2007). The dynamo benchmarks defined by Christensen et al. (2001) serve as test cases for the various methods; an update can be found in Wicht et al. (2009).

\section{Convective Flow Dynamics}

Am important consequence of the strong Coriolis force in planetary dynamo regions is formulated by the Taylor-Proudman theorem: The system seeks to minimize flow variations in the direction of the planetary rotation axis. A geostrophic flow is therefore generally two-dimensional and takes the form of convection columns that are aligned with the planetary rotation axis. In a spherical container, however, convective motions can not be strictly geostrophic since they will be diverted when meeting the boundaries. This gives rise to a secondary north-south meridional flow component. 

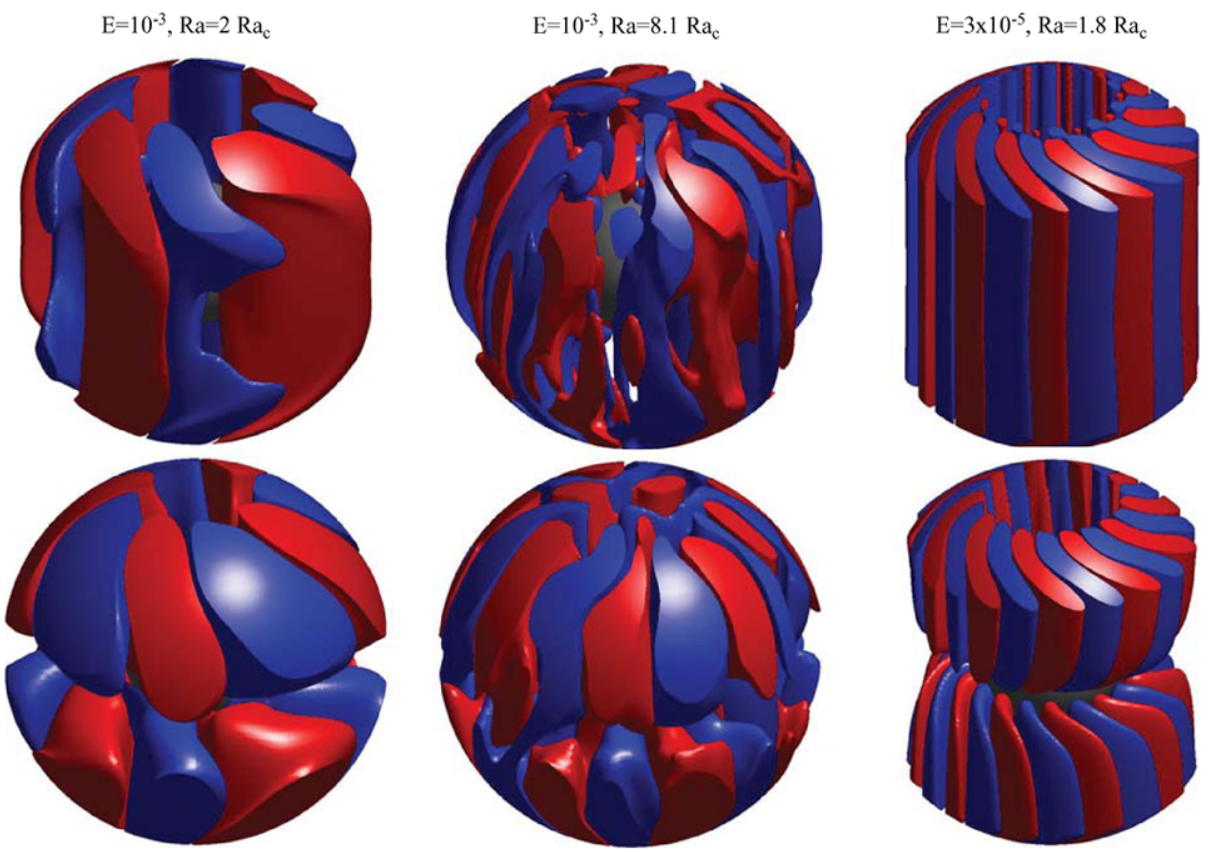

Fig. 2 Flow in non-magnetic convection simulations at different parameter combinations. The top row shows positive (red) and negative (blue) iso-surfaces of the axial vorticity, the bottom row shows positive (red) and negative (blue) iso-surfaces of the z-component of the flow. The Prandtl number is unity in all cases

Figure 2 illustrates the flow structure in non-magnetic convection simulations at the Ekman numbers $\mathrm{E}=10^{-3}$ (left and middle) and $\mathrm{E}=3 \times 10^{-5}$ (right). Iso-surfaces of the axial vorticity $\hat{\mathbf{z}} \cdot(\nabla \times \mathbf{U})$ visualize the convective columns that dominate the dynamics in all cases. The stronger Coriolis force influence clearly promotes a more two-dimensional geostrophic configuration in the lower Ekman number model. Increased buoyancy forces and nonlinear interactions, on the other hand, lead to a less geostrophic flow at higher Rayleigh numbers. The lower panels in Fig. 2 show the axial flow component ( $z$-component) to visualize the meridional circulation which is directed away from the equatorial plane in convective columns that rotate in prograde direction (cylones, red) but converges at the equatorial plane in retrograde rotating columns (anti-cyclones, blue). Thus, the $z$ component of the helicity $h=\mathbf{U} \cdot(\nabla \times \mathbf{U})$ has predominantly one sign in the northern hemisphere and the opposite sign in the southern hemisphere. Helicity is known to play an important role in the dynamo process and we will outline in Sect. 5.1 how its large scale coherence is responsible for creating a dipole dominated magnetic field.

Figure 2 demonstrates that the decrease of the Ekman number goes along with a smaller length scale. The reason becomes apparent when considering the linear onset of convection (Roberts 1968; Busse 1970): While the main part of the Coriolis force is balanced by pressure gradients, viscous forces must balance a remaining smaller part in order to facilitate convection (Zhang and Schubert 2000). As the Ekman number is lowered, the balance can only be maintained by further decreasing the length scale in order to keep viscous effects large enough. The convection sets in with a critical wave number $m_{c}$ that scales like $m_{c} \sim E^{-1 / 3}$ in the asymptotic limit for infinitely small Ekman number. The classical critical Rayleigh number $\mathrm{Ra}_{c}$, following definition (16), grows like $\mathrm{Ra}_{c} \sim E^{-4 / 3}$ because of 
the increasingly impeding action of the Coriolis force. For the two Ekman numbers illustrated in Fig. 2 the critical wave number grows from $m_{c}=4$ at $\mathrm{E}=10^{-3}$ to $m_{c}=10$ at $\mathrm{E}=3 \times 10^{-5}$ while the classical critical Rayleigh number increases from $\mathrm{Ra}_{c}=5.5 \times 10^{4}$ to $\mathrm{Ra}_{c}=2.8 \times 10^{6}$.

Close to onset, the convection is periodic in azimuth (according to $m_{c}$ ) and also symmetric with respect to the equator. When the Rayleigh number is increased beyond its critical value, the system undergoes a succession of symmetry breaking that is accompanied by a decrease in length scale and a growing complexity in time behavior. The azimuthal symmetry is lost first, then the equatorial symmetry. Solutions that are symmetric in azimuth simply drift around the rotation axis, but this simple time dependence is superseded by oscillatory and finally chaotic behavior once the azimuthal symmetry is broken. The second column in Fig. 2 shows a solution at $\mathrm{Ra}=4.5 \mathrm{Ra}_{c}$ with chaotic time behavior and broken azimuthal and equatorial symmetry. The flow remains, nevertheless, predominantly equatorially symmetric at the parameters explored in typical planetary dynamo simulations owed to the prevailing importance of the Coriolis force (Taylor-Proudman theorem). The succession of time dependencies is illustrated in Fig. 4.

Decreasing Ekman number and increasing Rayleigh number both promote primarily smaller azimuthal length scales. The length scale perpendicular to the rotation axis is much less effected and the convective columns therefore change from a rounder to a more sheet like shape. The length scale in the direction of the rotation axis also seems to vary only little over the Rayleigh numbers explored in typical dynamo simulations where the Coriolis force continues to impose a dominantly geostrophic structure.

The tangent cylinder is an imaginary boundary that is aligned with the rotation axis and touches the inner-core equator. It separates the shell into three regions with distinct dynamical behavior: the two regions inside the tangent cylinder above and below the inner core and the region outside the tangent cylinder. The columnar convection discussed above is restricted to the latter region. Inside the tangent cylinder, the convective motions are more effectively prohibited by the Taylor-Proudman effect since gravity roughly acts in the direction of the rotation axis here. Consequently, the convection starts somewhat later at Rayleigh numbers which are a few time $\mathrm{Ra}_{c}$ and it takes the form of local plumes or upwellings rather than columns (Tilgner and Busse 1997). The associated lateral temperature differences can drive strong thermal winds inside the tangent cylinder (Aubert 2005; Sreenivasan and Jones 2006). Thermal winds obey a balance between the curl of the Coriolis force and the buoyancy term in the Navier-Stokes equation (6):

$$
2 \frac{\partial \mathbf{U}}{\partial z}=-\operatorname{Ra}^{\star} \frac{r}{r_{o}} \nabla \times(T \hat{\mathbf{r}}) .
$$

Thermal zonal winds are thus driven by longitudinal temperature differences and show a characteristic z-variation which is distinctly non-geostrophic.

Figure 9a illustrates the zonal flow structure typical for convection simulations at higher Rayleigh numbers. Outside the tangent cylinder, Reynolds stresses drive a retrograde inner and a basically prograde outer flow (Christensen 2002; Heimpel et al. 2005; Aubert 2005). Thermal winds clearly dominate the region inside the tangent cylinder and are responsible for the weaker north south variation in the outer region. We will discuss the zonal flows further in comparison with the dynamo cases in Sect. 5.4.

The impact of inertial effects scales with the inverse of the Prandtl number. The above described scenarios apply for moderate Prandtl numbers of about one and mildly larger. For large Prandtl numbers, the bending of the columns and the associated Reynolds stresses become smaller. At Prandtl numbers below one, however, the Reynolds stress driven zonal 
winds can become so strong that the associated shear virtually stops the convection every now and then. This results in a particular time dependence called relaxation oscillation by Grote and Busse (2001). Also, the convection inside the tangent cylinder sets in at lower Rayleigh numbers for smaller Prandtl numbers. At very low Prandtl numbers, however, the dynamics is ruled by thermal inertial waves or pure inertial waves which have too short time scales, in the order of days, to affect the dynamo process (Zhang and Schubert 2000).

\section{Numerical Dynamo Solutions, an Overview}

\subsection{Fundamental Dynamo Mechanism}

The relatively simple structure of the benchmark dynamo (Christensen et al. 2001) allows to unravel the details of the dynamo process and to understand how it shapes the field at the top of the dynamo region. The magnetic field is produced in an $\alpha^{2}$ mechanism, a terminology that goes back to mean field dynamos (Sect. 2) and here simply means that the large scale poloidal and toroidal magnetic fields are created by the action of small scale flow on small scale magnetic field. This is likely the dominant process for all dynamo solutions presented here. An alternative is the $\alpha \omega$ mechanism where the toroidal field is created by shear in the zonal rotation $\omega$. It may, for example, be responsible for the field production in dynamo models with stress free boundary conditions that allow for larger zonal flows in the Kuang and Bloham or the Busse group models (Sect. 3.4).

The particular $\alpha^{2}$ mechanism presented here has been envisioned by Olson et al. (1999) and was later confirmed in a detailed analysis by Wicht and Aubert (2005) and Aubert et al. (2008b). Figure 3 shows radial magnetic fields at the outer boundary $r_{o}$ in the top panels and iso-surfaces of the axial vorticity along with magnetic fieldlines in the lower panels. The thickness of the fieldlines is proportional to the local magnetic field strength and their color indicates the field direction: red and blue stand for radially outward and inward pointing fields, respectively. The left column of Fig. 3 shows the benchmark II dynamo (model E3a) where the magnetic field production cycle can more clearly be discerned. The radial outflow between cyclonic (red) and anti-cyclonic columns (blue) grabs a north-south oriented fieldline around the equatorial plane and stretches it towards the outer boundary. This produces strong inverse radial field on either side of the equatorial plane that shows as prominent thick fieldlines in the foreground of Fig. 3. The pairwise inverse equatorial field patches often found at the outer boundary of many dynamo simulations are a result of this process.

The fieldlines are then wrapped around the anti-cyclones and are subsequently advected and stretched in northward and southward direction on both sides of the equator, respectively. Responsible for the latter action are the secondary meridional or axial flows discussed in Sect. 4 which are directed away from the equatorial plane in anti-cyclones but converge at the equatorial plane in cyclones. This is where the $z$-component of the helicity comes into play. The coherent separation of the opposing fields that largely cancels in the equatorial region is vital for producing the global dipole field. The imaginary cycle ends with another north-south oriented fieldline that amplifies the starting line and thereby compensates Ohmic decay. Since the anti-cyclonic columns are the more active part in the above described process the resulting magnetic structure has been called a magnetic anti-cyclones by Aubert et al. (2008b).

Flows converging where cyclones come close to the outer boundary further shape the magnetic field by advectively concentrating the background dipole field. The location of 

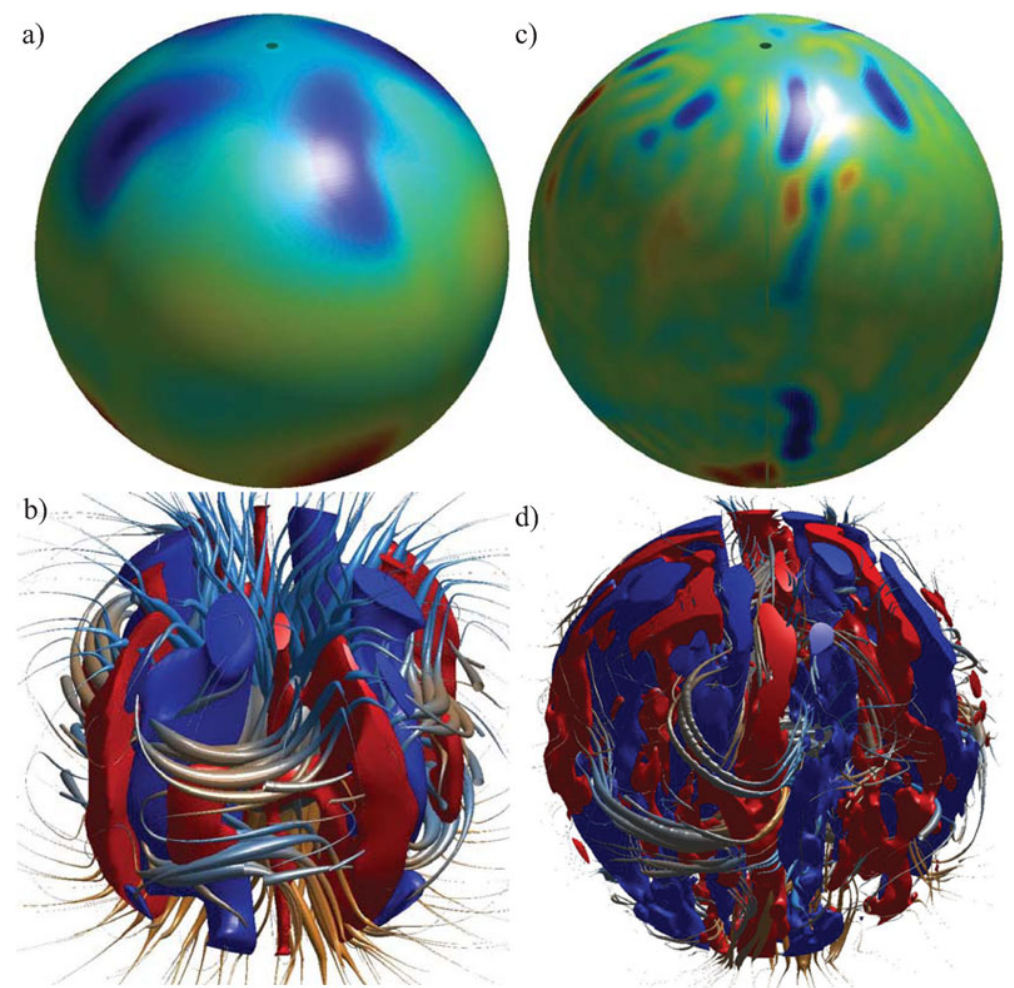

Fig. 3 The top panels (a) and (c) show snapshots of the radial magnetic field at the outer boundary $r_{O}$ for the model E3a (dynamo benchmark II) and model E3b which undergoes magnetic field reversals. Red and blue indicates radially outward and inward field, respectively. The lower panels show positive (red) and negative (blue) iso-surfaces of the axial vorticity along with magnetic fieldlines to visualize the dynamo process. The thickness of the fieldlines is proportional to the local magnetic energy, the color indicates the radial direction

the resulting mid to high latitude normal flux spots on the outer boundary roughly indicates where the convective columns touch the tangent cylinder and may be used as a proxy for the inner core size (Stanley et al. 2007). Inside the tangent cylinder, the magnetic field is weakened by advective processes due to the meridional circulation that is directed away from the pole and converges into the cyclonic columns.

The right panels in Fig. 3 show the configuration during a stable polarity epoch in the reversing higher Rayleigh number model E3b. While the structure is significantly more complex and more difficult to interpret than in the benchmark, similar features can nevertheless be discerned: (1) inverse field associated with radial outflows in the equatorial region, (2) the stretching of fieldlines towards the equator in cyclones but towards the poles in anticyclones, (3) the concentration of normal polarity field where flows converge into cyclones, and (4) the weakening of magnetic field inside the tangent cylinder. A feature typical for dynamos at larger Rayleigh numbers is the production of stronger azimuthal toroidal magnetic field in the outer parts of the equatorial plane. A prominent example can seen in the left equatorial region in panel (d) of Fig. 3. Strong toroidal field is produced by azimuthal flows mostly associated with outward stretching flow cyclones. This toroidal field together with the concentrated normal polarity field patches and the equator ward stretching of field- 
lines associated with cyclonic columns form the magnetic cyclones introduced Aubert et al. (2008b).

\subsection{Dynamo Regimes}

Figure 4a illustrates how the properties of a numerical dynamo model depend on Rayleigh and magnetic Prandtl number in a standard model. Ekman number and Prandtl number are kept fixed at $\mathrm{E}=10^{-3}$ and $P=1$, respectively. The diagram includes the benchmark case II (Christensen et al. 2001) which has been marked by a large square in panel (a) and is listed as model E3a in Table 1. Increasing the Rayleigh number is equivalent to increasing the thermal driving while larger magnetic Prandtl numbers represent larger electrical conductivities. Increasing either parameter can contribute to pushing the magnetic Reynolds number beyond the critical value for onset of dynamo action; there is therefore a certain tradeoff between the two.

The different symbols in Fig. 4 indicate the time behavior. Solutions that simply drift in azimuth (squares) are superseded by oscillating (triangles) and finally chaotic solutions (circles) when the Rayleigh number is increased. The time dependence basically follows the behavior of the purely convective solutions (grey symbols) including the associated symmetry breaking. Exceptions can be found at low Rayleigh numbers where the drifting and oscillatory solutions are replaced by chaotic solutions for larger magnetic Prandtl numbers. The highly symmetric solutions with simple time dependencies occupy only a very small fraction of the parameter space at low Rayleigh numbers and thus seem of little importance for planetary dynamos.

The colored contours in Fig. 4a show the dipolarity measure D, the relative rms dipole field strength at the top of the dynamo region. When the Rayleigh is increased, the dynamo
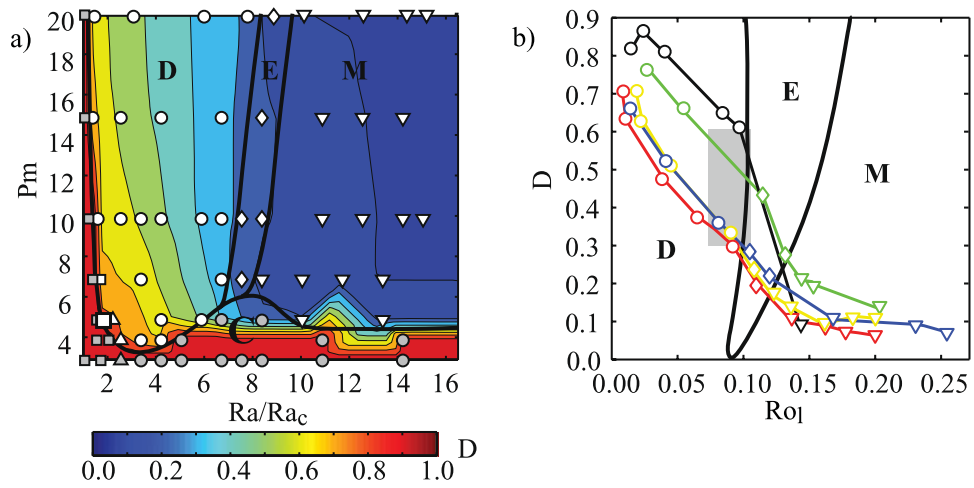

Fig. 4 Regime diagrams that illustrates the transition from stable dipole dominated (regime D) to constantly reversing multipolar dynamos (regime $\mathbf{M}$ ). Earth-like rarely reversing models (regime E) can be found at the transition. Panel (a) is based on standard models with $\mathrm{E}=10^{-3}$. Grey symbols mark non-magnetic convective solutions (regime C). Different symbols code the time dependence: squares = drifting, upward pointed triangles $=$ oscillatory, circles $=$ chaotic, diamonds $=$ Earth-like rarely reversing, downward pointed triangles $=$ constantly reversing. Panel (b) shows the dependence of the dipolarity measure D on the local Rossby number $\mathrm{Ro}_{\ell}$ for five different dynamo models. Blue and red: models from panel (a) with Pm $=10$ and $\mathrm{Pm}=20$, respectively; yellow: identical parameters to the blue model but with chemical boundary conditions; green: $\mathrm{E}=3 \times 10^{-4}, \mathrm{Pm}=3$, chemical boundary conditions; black: $\mathrm{E}=3 \times 10^{-5}$, Pm $=1$, fixed temperature conditions. The Prandtl number is unity in all cases. Chemical convection refers to codensity models that force the codensity flux to vanish at the outer boundary while a fixes codensity is used at the inner boundary (Kutzner and Christensen 2002). The grey rectangle shows the range of estimated values for the geodynamo (Wicht et al. 2009) 


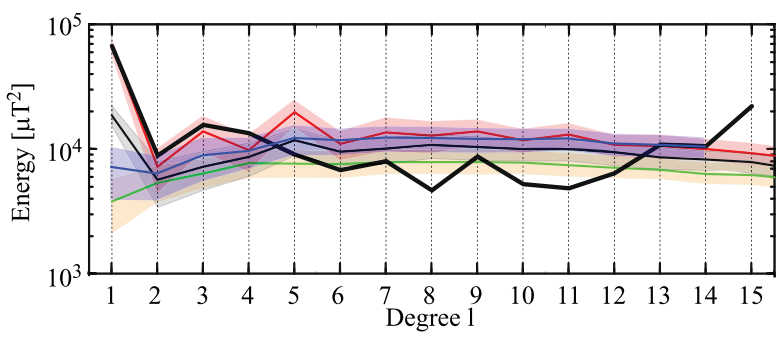

Fig. 5 Time averaged magnetic energy spectra for three dynamo simulations at $\mathrm{E}=3 \times 10^{-4}, \operatorname{Pr}=1$, and $\mathrm{Pm}=3$ that use chemical boundary conditions. These models contribute to the green curve in Fig. 4 and show solutions in regime $\mathbf{D}\left(\operatorname{Ro}_{\ell}=0.07\right.$, red line $)$, in regime $\mathbf{M}\left(\operatorname{Ro}_{\ell}=0.13\right.$, green line $)$, and in regime $\mathbf{E}$ $\left(\mathrm{Ro}_{\ell}=0.21\right.$, black and green lines $)$. The black line depicts a stable polarity epoch while the green line depicts a transitional epoch of this reversing model. The time averaging has been performed over periods equivalent to several $10000 \mathrm{yr}$ in each case. Transparent colored bands in the with of the standard deviation illustrate the time variability. The thick black line shows the spectrum for the geomagnetic field model POMME for the year 2000 (Maus et al. 2006)

changes from a dipole dominated regime $\mathbf{D}$ to a multipolar regime $\mathbf{M}$ where the dipole component has lost its special character (Kutzner and Christensen 2002; Christensen and Aubert 2006; Olson and Christensen 2006; Wicht et al. 2009). While the dipole never reverses in regime $\mathbf{D}$ it continuously switches polarity in regime $\mathbf{M}$. Earth like reversing behavior (regime $\mathbf{E}$ ), where the dipole still dominates in a time mean sense and polarity transitions, are relatively rare events can be found at the transition. We assume that a model qualifies for this regime when the time averaged dipolarity $\mathrm{D}$ is larger than 0.2 and the magnetic pole spends less than a tenth of the time further than $45^{\circ}$ degree away from the closest geographic pole. This definition is somewhat arbitrary (Wicht et al. 2009) but nevertheless demonstrates that the Earth-like regime occupies only a small part of the parameter space.

Figure 5 compares time averaged magnetic energy spectra of cases in regimes $\mathbf{D}, \mathbf{E}$, and $\mathbf{M}$ for $\mathrm{E}=3 \times 10^{-4}$ with the spectrum of a geomagnetic field model for the year 2000 (Maus et al. 2006). The spectra show the magnetic energy carried by the spherical harmonic contributions of degree $l$ at the outer boundary (cmb). The simulations have been scaled by assuming realistic values for $\Omega, \bar{\rho}, \mu$, and $\lambda$ (Sect. 3.3) to facility the comparison with the geomagnetic model. Spectra during the transitional periods in regime $\mathbf{E}$ show the same low dipole contribution as those in regime M. Kutzner and Christensen (2002) thus conclude that the models in regime $\mathbf{E}$ briefly venture into the configuration that is assumed more or less constantly for the larger Rayleigh numbers cases in regime $\mathbf{M}$.

Kutzner and Christensen (2002) and Christensen and Aubert (2006) demonstrate that this basic regime diagram also holds for smaller Ekman numbers with two interesting differences. First, as the Ekman number is decreased the boundary between the dipolar and the multipolar regimes moves further out to higher values of $\mathrm{Ra} / \mathrm{Ra}_{c}$. Second, the critical magnetic Prandtl number $\mathrm{Pm}_{c}$ at which dynamo action is still possible decreases. For the simulations at $\mathrm{E}=10^{-3}$ shown in Fig. 4 no dynamos are found below $\mathrm{Pm}_{c}=4$ (all magnetic field decayed at $\mathrm{Pm}=3$ ); the values goes down to $\mathrm{Pm}_{c}=0.1$ at $\mathrm{E}=10^{-5}$ (Christensen and Aubert 2006). This phenomenon has been examined by Takahashi and Matsushima (2006) who suggest a connection with the magnetic Reynolds number. They argue that an effective magnetic Reynolds number could be considered which accounts for the different length scales in the induction equation:

$$
\mathrm{Rm}_{E}=\frac{\mathrm{U} L^{2}}{\lambda \ell}=\mathrm{Rm} \frac{L}{\ell} .
$$


This definition assumes a dipole dominated magnetic field with length scale $L$ and an $\alpha$ effect that operates on the flow length scale $\ell$ (22). Since $\ell$ decreases with the Ekman number one can get away with smaller values of $\mathrm{Rm}$ and still maintain the necessary effective magnetic Reynolds number for dynamo action. Smaller values of Rm translate into larger magnetic diffusivities or smaller magnetic Prandtl numbers for fixed values of the flow amplitude $U$.

Christensen and Aubert (2006) argue that the transition from the dipolar to the multipolar regime should more appropriately by discussed in terms of the local Rossby number $\mathrm{Ro}_{\ell}$ introduced in (21). They examined a suite of dynamo simulations at various parameter combinations and found that the transition always takes place around $\mathrm{Ro}_{\ell} \approx 0.12$ (also see Christensen [doi:10.1007/s11214-009-9553-2], this issue). This is also true for all the models presented here and has been confirmed for many others (Olson and Christensen 2006; Takahashi et al. 2008a; Wicht et al. 2009). The examination of Christensen and Aubert (2006) was restricted to dynamos with fixed temperature boundary conditions but also seems to apply to other driving modes and thermal boundary conditions (Olson and Christensen 2006; Wicht et al. 2009; Aubert et al. 2009) with some exceptions that we discuss at the end of this chapter. Figure $4 \mathrm{~b}$ compares the respective regime diagram for five dynamo models that vary in thermal boundary condition, magnetic Prandtl number, and Ekman number. Only the Ekman number seems to have a significant impact here: The transition steepens when the Ekman number is decreased. At lower Ekman numbers the solutions remain very dipolar right up to the transitions to the multipolar regime. The $\mathrm{D}$ values only become Earth-like in regime $\mathbf{E}$ (Wicht et al. 2009). The heat-flux based scaling suggested by Christensen and Aubert (2006) places Earth at about $\mathrm{Ro}_{\ell} \approx 0.09$ (Christensen [doi:10.1007/s11214-009-9553-2], this issue) which is nicely compatible with the fact that numerical simulations show Earth-like reversals at comparable $\mathrm{Ro}_{\ell}$ values.

The dependence on the local Rossby number indicates that inertia, or more precisely the nonlinear advection term in the Navier-Stokes equation (6), plays an important role for the transition from dipolar to multipolar dynamos. The exact reason, however, is not understood nor do we know why the transition should happen at this particular value of $\mathrm{Ro}_{\ell}$. Wicht et al. (2009) suggest that the mixing due to nonlinear advection counteracts the ordering influence of the Coriolis force. As a consequence, the dynamo looses its ability to maintain a globally correlated large scale field. We come back to this point in Sect. 6 . The value of about $\mathrm{Ro}_{\ell} \approx 0.09$ suggested for Earth questions the common conclusion that inertia in negligible in planetary dynamo regions. Using the relation $\mathrm{Ro}_{\ell}=\mathrm{Ro} L / \ell$ to estimated the involved length scale renders $\ell=4 \times 10^{-5}$ which is equivalent to $100 \mathrm{~m}$ in Earth's core. It is hard to conceive that the flow on such a small length scale should influence the dipole behavior. Clearly, more research is need to elucidate the meaning of the local Rossby number.

Sreenivasan and Jones (2005) explore what happens when Prandtl number and magnetic Prandtl number are varied while Rayleigh number and Ekman number are kept fixed. Decreasing Pr increases the importance of inertia in the system and therefore offers an alternative route into the regime where inertia play a more significant role. The study by Sreenivasan and Jones (2005) confirms that the growing influence of inertial forces gradually decreases the symmetry of the solutions and leads from dipole dominated magnetic fields to configurations where the dipole component is significantly less important. Their simulations were not run for long enough to establish whether these latter solutions would actually reverse.

Some authors report deviations from the above described regime scenario. Li et al. (2002) and Nishikawa and Kusano (2008) present Kageyama type models where magnetic field reversals set in at relatively low Rayleigh numbers when the magnetic Prandtl number is 
increased (see also McMillan and Sarson 2003). This is at odds with the minor influence illustrated in Fig. 4 where a rise in Pm can provoke a more complex time dependence and a less dipolar field but never causes reversals. Both group of authors employ parameters similar to the ones explored in Fig. 4 but retain weak compressibility effects instead of using the Boussinesq approximation. Whether this really explains the differences remains to be explored.

The work of Simitev and Busse (2009) offers another interesting view point. Like Sreenivasan and Jones (2005) these authors explore the Prandtl number dependence of their Busse group dynamo model. They find dipole dominated stable solutions for larger Prandtl numbers, where inertia is less important, and reversing multipolar solutions for small Prandtl numbers. For Prandtl numbers around $\operatorname{Pr} \approx 1$, however, both types of solutions can be found depending on the starting condition of the simulation. A similar case is reported by Christensen and Aubert (2006). The possible coexistence of different attractors is a common property of non-linear systems and needs to be explored further in the dynamo context.

Under certain conditions, non-dipolar dynamos may already be preferred at low Rayleigh numbers right at the onset of dynamo action. For example, Kutzner and Christensen (2002) find multipolar solutions for a standard model which, however, is driven exclusively by internal heat sources. Grote et al. (1999) and Grote and Busse (2000) report Busse group models that are dominated by the magnetic quadrupole term or mainly operate in one hemisphere. These cases are to some degree driven by volumetric buoyancy sources which suggest that this driving type promotes non-dipolar dynamos. Aubert et al. (2009), on the other hand, find clearly dipole dominated purely volumetric driven dynamos. The reason for the differences remains unclear but the existence of two parallel attractors once more offers a possible explanation (Simitev and Busse 2009). A more extensive discussion on non-dipolar dynamos can be found in Stanley and Glatzmaier [doi:10.1007/s11214-009-9573-y], this issue).

\subsection{Magnetic Field Structure}

Figure 6 demonstrates how the magnetic field structure changes when the Ekman number is decreased with representative snapshots. Model E3b is the larger Ekman number model (E = $10^{-3}$ ) we depicted to explain the dynamo mechanism (Fig. 3). Models E5b, E5F, and E5c share the low Ekman number $\mathrm{E}=3 \times 10^{-5}$ as well as identical Prandtl and magnetic Prandtl numbers $(\operatorname{Pr}=1, \operatorname{Pm}=1)$. Model E5b has a local Rossby number of $\operatorname{Ro}_{\ell}=0.97$ where reversals can be expected, but we did not observe any such event in the limited time we where able to compute. The larger Rayleigh number in Model E5c increases the local Rossby number to $\mathrm{Ro}_{\ell}=0.14$; consequently the dipole is weak and reverses frequently. Model E5F has a lower Rayleigh number that places it safely into the dipole dominated regime at $\mathrm{Ro}_{\ell}=$ 0.065 and is the only case where stress free boundary conditions are employed here. Finally, model E6 has the lowest Ekman number of $E=3 \times 10^{-6}$ and a moderate Rayleigh number. The dipole component is rather strong and the local Rossby number small at $\operatorname{Ro}_{\ell}=0.023$. We imposed a four fold azimuthal symmetry in model E6 in order to reduce the numerical costs. Parameters and properties of all cases are listed in Table 1.

The left column of Fig. 6 shows the radial field at the outer boundary. The comparison between models E3b and E5b once more reveals the smaller scales found at lower Ekman numbers but also shows many similarities: (1) The strong normal polarity patches close to where the tangent cylinder touches the outer boundary, (2) the weakened field inside the tangent cylinder, (3) inverse field patches inside the tangent cylinder, and (4) pairwise inverse field patches around the equator. When the Ekman number is reduced from $\mathrm{E}=3 \times 10^{-5}$ in model $\mathrm{E} 5 \mathrm{~b}$ to $\mathrm{E}=3 \times 10^{-6}$ in model $\mathrm{E} 6$, however, interesting differences appear. The normal 

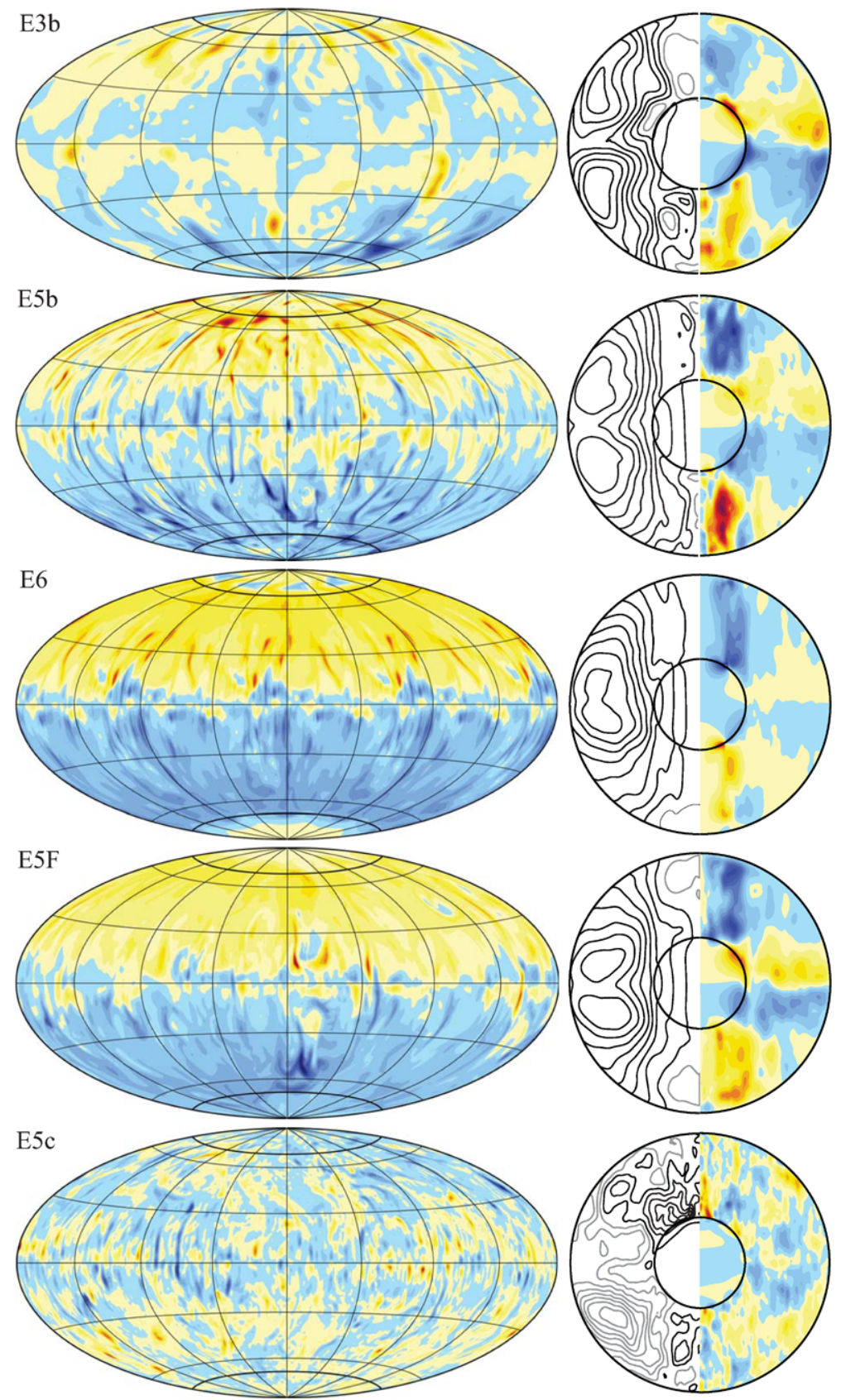

Fig. 6 Radial magnetic field at the outer boundary (left panels), axisymmetric magnetic field lines (right panels, left half) and axisymmetric toroidal field (right panels, right half) for five dynamo models. See Table 1 for parameters. Blue (yellow/red) colors refer to radially inward (outward) or retrograde (prograde) field directions, respectively. The field direction is clockwise (counter-clockwise) along black (grey) fieldlines 
polarity field is more evenly distributed over both hemispheres, respectively, and normal polarity patches also dominate at mid to lower latitudes. The pairwise inverse field patches around the equator are now limited to a thin latitude band. The stress free model E5F shows a very similar configuration. The Coriolis force still seems to be too little influential at larger Ekman numbers. At model E5b either the stronger impact of inertial forces due to the larger Rayleigh number and/or the somewhat larger viscosity may still prevent the lower latitude field to be more Earth-like. The former reason seems more likely, since the viscous boundary friction is already quire low. Dynamo simulations by Takahashi et al. (2008a) show a similar transition at comparable Ekman numbers.

Figure 7 compares the filtered outer boundary field in models E5b and E5F with the geomagnetic field model GUFM for the epoch 1990 (Jackson et al. 2000). The filter simulates the effect of Earth's crustal magnetization that screens the internal field beyond spherical harmonic degree $l=12$. Both numerical models agree with the geomagnetic field in several properties: the dipole dominance, the typical high latitude normal polarity patches, the weakened or inverse field inside the tangent cylinder, and the presence of inverse patches in the equatorial region. The strong normal polarity patches close to the equator, that are evident in Fig. 7, seem to be typical for the historic geomagnetic field (Jackson 2003; Jackson and Finlay 2007). Whether they also form an important characteristic of the fundamental geodynamo process and would prevail in a long time scale average is unknown. The low latitude normal patches are much weaker in our models E6 and E5F and inverse patches are less abundant than in the historic geomagnetic field. Whether this changes at larger Rayleigh numbers where reversals can be expected remains to be explored. The strong nor-

Fig. 7 Comparison of the radial magnetic field at the core-mantle boundary for the 1990 GUFM model (Jackson et al. 2000) and filtered snapshots at the outer boundary off models E5b and E5F. Figure 6 shows the full numerical resolution for both numerical models. We have inverted the field direction here, to ease the comparison with the geomagnetic model
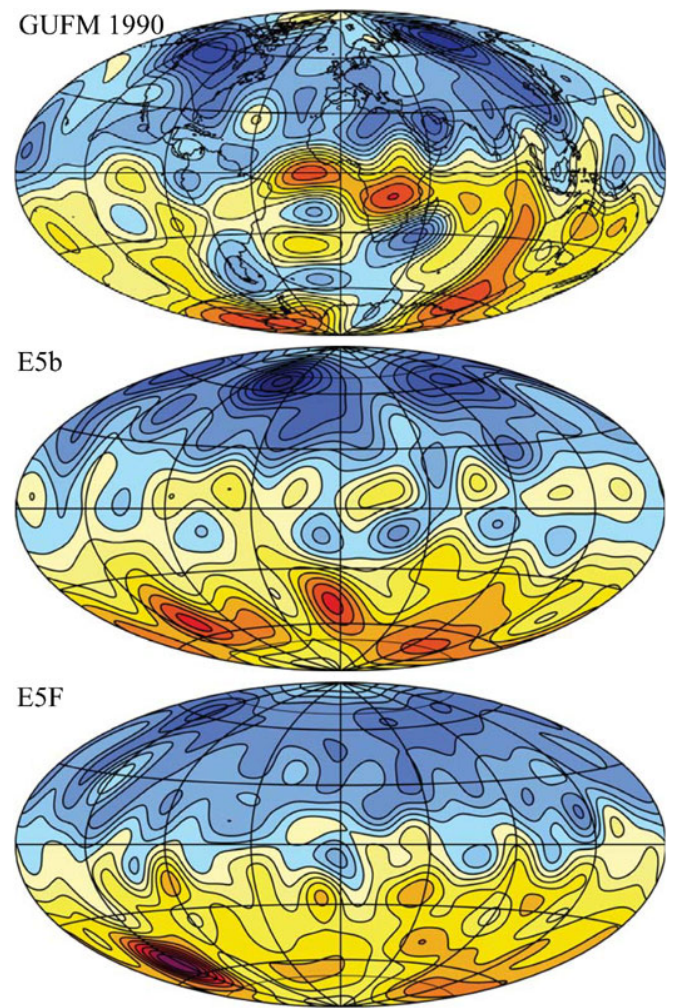
mal polarity patches close the tangent cylinder still remain prominent in the filtered outer boundary field in models E6 and E5F (see Fig. 7 for the latter case). Close-by inverse field patches evident in the full resolution fields shown in Fig. 6 reduce the impact of the low to mid latitude normal patches in the filtered field version.

The change in the dynamo mechanism responsible for the difference in field geometry in unknown. Except for the multipolar model E5c the axisymmetric poloidal fieldlines and the axisymmetric azimuthal toroidal fields shown in Fig. 6 are remarkable similar. Inverse fieldlines around the equatorial plane seem a common feature but become weaker for lower Ekman numbers which is likely a condition for the prevailing normal polarity $\mathrm{cmb}$ field at lower latitudes. The strong axisymmetric azimuthal toroidal field found inside the tangent cylinder in many dynamo models at larger Rayleigh numbers is produce by the thermal winds via an $\omega$ effect.

\subsection{Flow Structure}

Figure 8 illustrates the flow structures that correspond to the magnetic field snapshots shown in Fig. 6. The comparison of flow and magnetic field structures for model E3c reveals that the latter is of smaller scale. This is owed to the larger magnetic Prandtl number of $\mathrm{Pm}=10$ in this model which means that the viscous diffusivity is an order of magnitude larger than the magnetic diffusivity. Figure 8 once more demonstrates that the convective features become progressively thinner and more sheet-like as the Ekman number is decreased. However, the sheets seem to break into smaller entities in the high Rayleigh number multipolar model E5c.

It is difficult to assess how the magnetic field changes the flow in order to saturate its growth. A simple primary effect is that the magnetic field decrease the rms flow amplitude $U$. A comparison between the convective columns in Fig. 2 and Fig. 3 also reveals that the cyclones are much smaller than the anti-cyclones in the presence of a magnetic field. This is due to the fact that the fieldlines tend to pass directly through the cyclones but stay more clear of the anti-cyclones (Aubert and Wicht 2004). However, the difference seems to vanish at larger Rayleigh numbers and smaller Ekman numbers. More research is required to elucidate the true saturation mechanism which may actually be quite subtle (Jones 2000).

Scenarios of magnetic field saturation are sometimes discussed in connection with a runaway growth of the magnetic field and the jump from a weak field to a strong field branch. On the weak field branch, the Lorentz force is too small to balance the Coriolis force significantly. Suppose however, that a field strength fluctuation leads to a slightly better cancelation between Lorentz and Coriolis force. This would somewhat alleviate the viscous force from balancing the Coriolis force to facilitate convection. The flow could assume a larger scale and would become more vigourous. This in turn produces a yet stronger magnetic field resulting in a runaway that ends on the strong field branch where the magnetostrophic balance is established at an Elsasser number of order one.

The promoting effects of magnetic fields on convection have extensively been explored in magnetostrophic models where the magnetic field is imposed rather than produced by a dynamo effect (see Wicht et al. 2009, for a recent overview). The change in flow length scale and the runaway growth have also been observed in simplified selfconsistent dynamo simulations that employ Cartesian geometries (Rotvig and Jones 2002; Stellmach and Hansen 2004). These simulations suggest that the Ekman number has to be small enough for these effects to be significant, probably as low as $\mathrm{E} \leq 10^{-5}$ (Stellmach and Hansen 2004). Though today's self-consistent spherical dynamo models have reached Ekman numbers as low as $\mathrm{E}=10^{-6}$ the predicted large scale change and the 

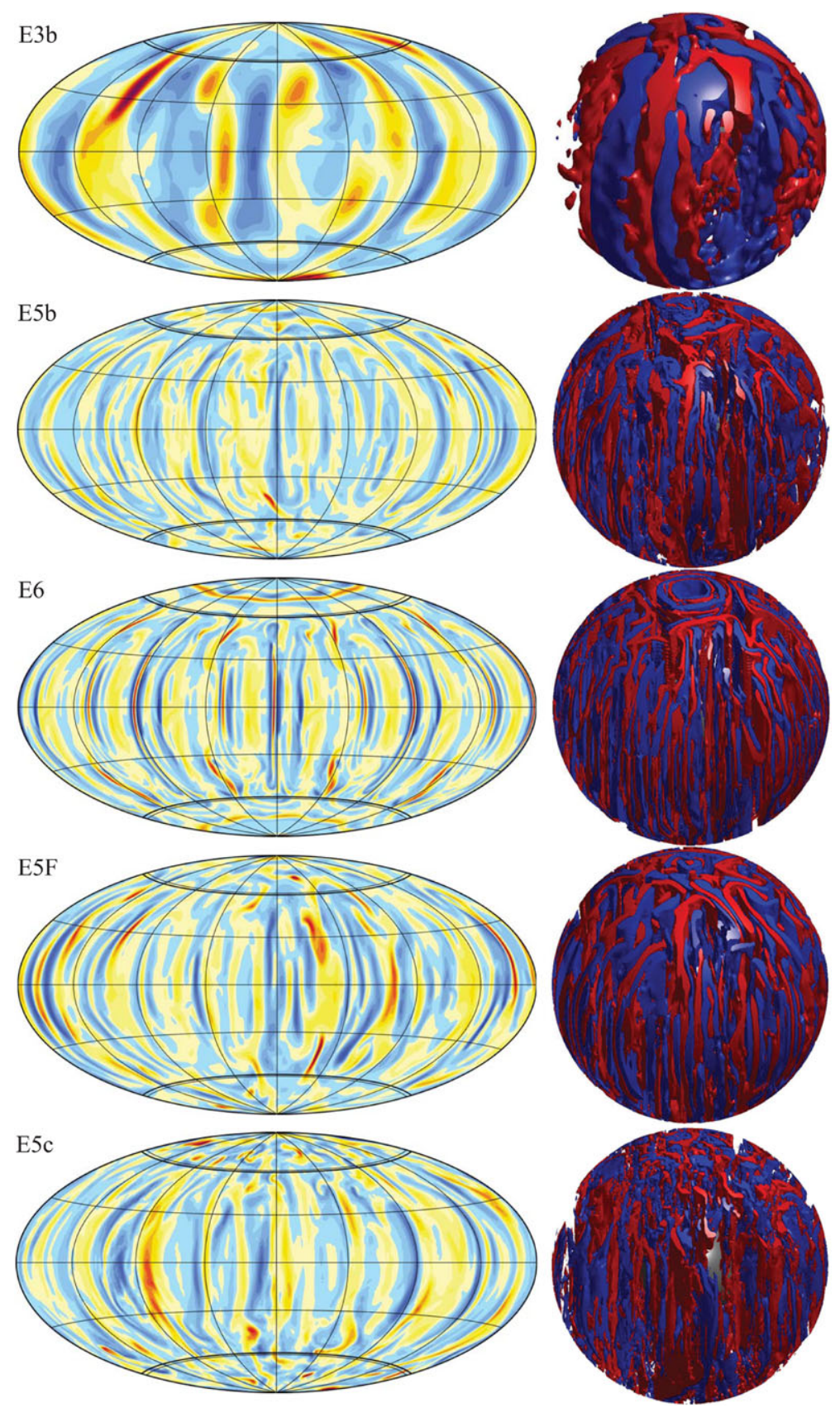

Fig. 8 Radial flow at mid shell $r=r_{i}+\left(r_{o}-r_{i}\right) / 2$ (left) and iso-surfaces of axial vorticity (right) for the models depicted in Fig. 6. Yellow/red (blue) contours stand for radially outward (inward) radial flows. Red (blue) iso-surfaces depict prograde (retrograde) rotating features 

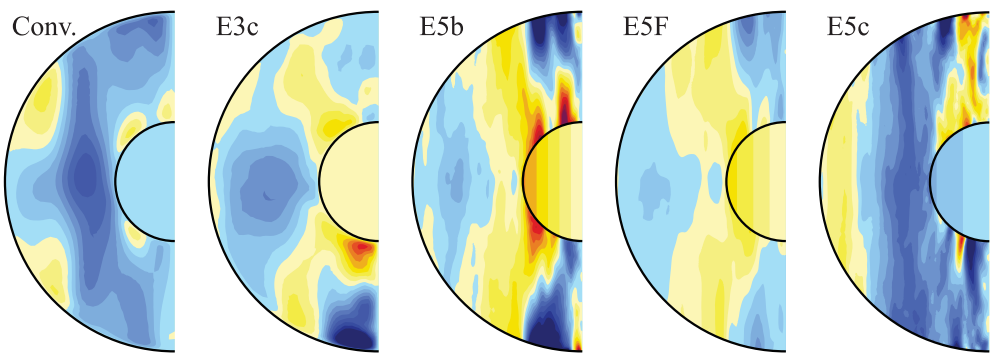

Fig. 9 Snapshots of he zonal flow in four different dynamo models and a non-magnetic convection simulation (Conv.) that uses the same parameters as model E3c. The contour steps are identical for the left tow panels and for the right three panels, respectively. The snapshots show typical large scale flow configurations. The smaller scale details may vary with time, in particular inside the tangent cylinder where the convective plumes become rather time dependent at larger Rayleigh numbers (cases E3c and E5c)

jump between two distinct branches has not been observed. Most if not all of these models would probably qualify as strong field cases. (Takahashi et al. 2008a) report that the mean flow scale $\ell$ (22) increases by only $20 \%$ when comparing a dynamo and a nonmagnetic simulation at $\mathrm{E}=10^{-6}$. The rather weak influence on the flow scale may be attributed to the fact that dynamos tend to adopt a configuration where the interaction between shear and magnetic field is minimized (Aubert and Wicht 2004; Aubert 2005; Jones 2007): the fieldlines wrap around the convective features rather than passing through them and mostly arrange themselves perpendicular to the shear which minimizes their interaction with the flow.

Figure 9 shows snapshots of the zonal flow that illustrate typical configuration in several numerical models. The leftmost panel depicts a non-magnetic case with the same parameters as the large Ekman model E3c. The comparison between the zonal flow in these two cases reveals a significant difference outside the tangent cylinder. Here, Reynolds stresses rule in the non-magnetic case while thermal winds dominate in the magnetic case. This effect has first been reported by Aubert (2005) who finds that the zonal components of Coriolis and Lorentz force balance to a good degree in dynamo models. The associated relaxation of the Taylor-Proudman theorem allows an increased heat transport in the equatorial region and inside the tangent cylinder. The resulting temperature gradients are responsible for the stronger thermal winds that dominate the zonal flow in dynamo simulations. The comparison of the zonal flows for models E5b and E5F in Fig. 9 shows that the main differences can be found inside the tangent cylinder where the higher Rayleigh number in the former model drives stronger thermal winds. Outside the tangent cylinder, the solutions are remarkably similar. We have analyzed the viscous forces acting on the zonal flows in model E5b and find that the viscous drag at the boundaries is comparable to the bulk friction. This may explain the minor differences between the stress free and the rigid boundary model.

The last panel in Fig. 9 shows the zonal flow in the larger Rayleigh number multipolar case E5c. The zonal flow is back to a Reynolds stress influenced configuration outside the tangent cylinder which suggests that the Lorentz force less efficiently balances the Coriolis force when the dipole component is weak. Inside the tangent cylinder, the structure is more complex. Possibly, Reynolds stress effects (Heimpel et al. 2005) and thermal wind effects are now at work here. The high latitude retrograde flow found close to the outer boundary in the dipole dominated cases is also a typical feature in core flow models derived from geomagnetic secular variation data (Eymin and Hulot 2005). Aurnou et al. (2003) and Aubert 
(2005) show that the amplitude of these flows can be scaled to Earth-like values based on the heat flux through the outer boundary as discussed in Sect. 3.3.

\section{Reversals Mechanisms}

Magnetic field reversals are the most drastic changes in the geomagnetic field. Paleomagnetic data quantify several of their properties but provide essentially no information about their causes and internal dynamics. Numerical dynamo models try to fill this void with extensive parameter studies and detailed analysis of simulated polarity transitions. We have outlined in Sect. 5.2 that many dynamo simulations show reversals when the convection is driven strongly enough to make the inertial forces sufficiently influential which seems to happen around a local Rossby numbers of $\mathrm{Ro}_{\ell} \approx 0.1$.

At which parameters reversals set in exactly is hard to determine numerically. Since the likelihood for reversals seems to increase with the local Rossby number (Wicht et al. 2009) and the duration of stable polarity epochs varies stochastically, models at lower $\mathrm{Ro}_{\ell}$ values may simply be categorized as non-reversing because the simulated time span is too short. Wicht et al. (2009) explored several dynamo models at $\mathrm{E}=10^{-3}$ and $\mathrm{Pm}=10$ where the computation of extremely long time spans could be afforded. No reversal was found during more than 140 magnetic diffusion times (equivalent to more than $17 \mathrm{Myr}$ for Earth) in a model with $\mathrm{Ro}_{\ell}=0.08$ while a model with $\mathrm{Ro}_{\ell}=0.10$ underwent 4 reversals during 87 magnetic diffusion times (equivalent to more than $10 \mathrm{Myr}$ for Earth). (Both cases entered Fig. 4.) This seems to support the rule of thumb that reversals start to appear around $\mathrm{Ro}_{\ell}=0.1$ but it can not be excluded that reversals just become extremely unlikely for smaller local Rossby numbers.

Several authors have observed that numerical reversal processes correlate with an increased equatorially symmetric magnetic field component ( $\mathrm{Li}$ et al. 2002; Takahashi et al. 2005, 2007, Nishikawa and Kusano 2008). This is typically accompanied by an increase of the equatorially asymmetric kinetic field which is required to convert equatorially asymmetric magnetic field into its symmetric counterpart. However, significant violations of either symmetry can already be observed for dynamos that never seem to reverse (Wicht et al. 2009). The breaking of equatorial and azimuthal symmetries are therefore necessary but not sufficient conditions. The same is true for the plume like convection inside the tangent cylinder that may also play a role in polarity transitions (Rotvig 2009).

Aubert et al. (2008b) have identified distinct magnetic features that are responsible for producing significant amounts of inverse magnetic field. These features are tied to flow upwellings or plumes and have therefore been named magnetic upwellings by these authors. They fall into two distinct categories: those that rise inside the tangent cylinder and those that prefer low to mid latitudes. The geomagnetic field shows inverse field patches in both regions.

Inside the tangent cylinder, the magnetic upwellings are connected to the convective plumes we briefly touched on in Sect. 4 (Aurnou et al. 2003; Aubert et al. 2008b). Sreenivasan and Jones (2006) show that the size and number of these plumes depends on the background magnetic field strength. Fewer but thicker plumes can be observed for stronger fields. This provides an interesting feedback mechanism whose role for the inverse field production has not yet been fully explored. The plumes inside the tangent cylinder can significantly reduce the background dipole field and tilt it to a limited extent.

More instrumental for field reversals, however, are the low to mid latitude magnetic upwellings. We have outlined in Sect. 5.1 that the outflows in the equatorial region produce a 


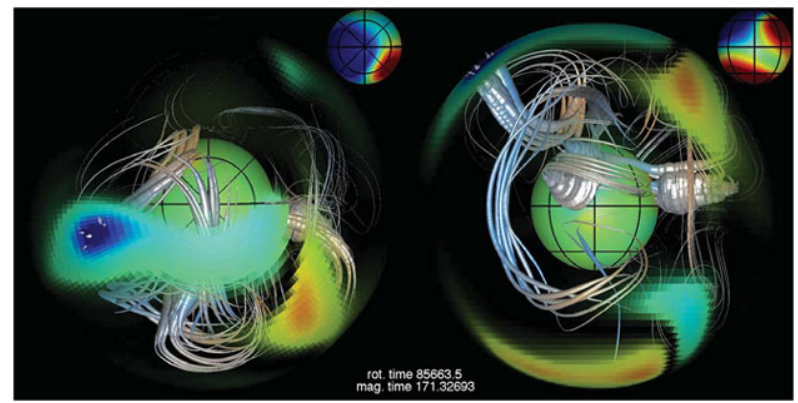

Fig. 10 Magnetic fieldlines during a reversal visualized with the DMFI tool (Aubert et al. 2008b). The thickness of the fieldlines has been scaled with the local magnetic energy. North-polar and side view are shown at left and right, respectively. The inner core is represented by the central sphere. Color coded radial magnetic field is shown on the inner core, the outer boundary (only dominant field patches), and at a level representing Earth's surface in the upper right corner of both panels. The snapshot depicts two magnetic upwellings in the northern hemisphere that have already created enough inverse field to significantly tilt the dipole. A magnetic-anticyclone can been seen in the left hemisphere

large amount of inverse field even in very simple dynamo models that never reverse. Here, inverse field of both polarities is produce at either side of the equatorial plane so that the effects largely cancel. In magnetic upwellings, the north/south coherence is obviously violated and inverse field is produced predominantly on one side of the equator (Aubert et al. 2008b; Wicht and Olson 2004). This corresponds to the loss of equatorial asymmetry in the magnetic field and of equatorial symmetry in the flow field described by some authors ( $\mathrm{Li}$ et al. 2002; Takahashi et al. 2007; Coe and Glatzmaier 2006; Nishikawa and Kusano 2008).

The typical time scale of the magnetic upwellings is about one kyr, but their duration, amplitude, and frequency seem to vary stochastically. Weaker magnetic field excursions can be caused by one magnetic upwelling of average amplitude. Full polarity reversals or strong global excursions with predominantly inverse field directions require a particularly fierce and long lasting upwelling to sufficiently cancel the normal polarity field. Alternatively, several upwellings can team up to perform the job. Both scenarios are unlikely which explains the rareness of these events. The simulations suggest that the cancelation of the normal polarity field is the essential process that enables reversals. It leaves a largely undecided field polarity and once the magnetic upwellings have ceased a subsequent restart of the 'normal' dynamo mechanism may produce field of either polarity, rendering the event a reversal or an excursion (Kutzner and Christensen 2002).

A detailed analysis of reversal mechanisms that considers the $3 \mathrm{~d}$ structure of flow and magnetic field is complex and has therefore been limited to a few cases, mostly at large Ekman numbers. The importance of magnetic upwellings for field reversals has most clearly been demonstrated at $\mathrm{E}=10^{-2}$ and $\mathrm{E}=2 \times 10^{-2}$ in the models by Wicht and Olson (2004) and Aubert et al. (2008b), respectively. Magnetic upwellings remain important at $\mathrm{E}=3 \times 10^{-4}$, but so far no detailed reversal analysis has been performed at this Ekman number (Aubert et al. 2008b). Takahashi et al. (2007) report that the inverse field in their model at $\mathrm{E}=2 \times 10^{-5}$ is part of a strong magnetic loop that correlates with a deformed convective column. Whether and how these features relate to magnetic upwellings remains unclear. Common to most reversal simulations is the importance of strong inverse magnetic field patches at low to mid latitudes in the beginning phase of a reversal (Driscoll and Olson 2009). This agrees with the findings in the paleomagnetic model for the Matuyama-Bruñes reversal by Leonhardt and Fabian (2007). 
Busse and Simitev (2008) describe a completely different reversal scenario which is based on a Parker dynamo wave: inverse field starts to grow on either side of the equatorial plane and propagates towards the poles (Parker 1955; Simitev and Busse 2005). A Parker wave is a purely magnetic linear oscillatory process that does not rely on any flow changes. Busse and Simitev (2008) find quasi oscillatory reversals in the toroidal magnetic field while the poloidal field varies to a much lesser extend. Only occasionally are the oscillations strong enough to cause a global excursion of field reversal. This explains their rareness in comparison to the proposed Parker wave period of $40 \mathrm{kyr}$ for Earth.

We have already discussed in Sect. 5.2 that the scaling based on the heat flux out of Earth core places the geodynamo at $\operatorname{Ro}_{\ell}=0.09$ and thus into the regime $\mathbf{E}$ where Earth like reversals are found in many simulations (Christensen and Aubert 2006; Olson and Christensen 2006). Should the weakly compressible models that seem to model reversals at smaller local Rossby numbers provide a vital alternative here (Li et al. 2002; Nishikawa and Kusano 2008; McMillan and Sarson 2003)?

We refer to Glatzmaier and Coe (2007) and Wicht et al. (2009) for a more detailed analysis of simulated reversals and a comparison with paleomagnetic findings.

\section{Torsional Oscillations}

Torsional oscillations are a specific form of flow variations that concern the rotation of geostrophic cylinders. Like the tangent cylinder, these cylinders are aligned with the planetary rotation axis. Their rotation is the only truly geostrophic flow component and also determines the total angular momentum of the fluid. Decadal variations in Earth's lengthof-day are commonly attributed to an exchange of angular momentum between core and mantle. This is strongly supported by the reasonable agreement between these variations and changes in the estimated core angular momentum (Jault et al. 1988; Jault and LeMouël 1989; Jackson et al. 1993; Jackson 1997; Jault 2003; Amit and Olson 2006). Torsional oscillations have a decadal time scale which is significantly shorter than the flow turnover time $t_{U} \approx 120 \mathrm{y}$ thought to be characteristic for the dynamo process. They may thus not only form an important part of the faster magnetic field variations but are also a likely cause for the decadal length-of-day variations (Braginsky 1970). Bloxham et al. (2002) suggest that torsional oscillations may also cause geomagnetic jerks, a rapid change in the geomagnetic secular variation signal on a yearly time scale. Moreover, they can provide valuable information in the internal dynamics of the dynamo region.

When integrating the azimuthal component of the Navier-Stokes equation over a geostrophic cylinder Coriolis force and pressure force drop out. Only Lorentz force, the nonlinear inertial term, and viscous effects therefore contribute to the acceleration of these cylinders. The latter two contributions are generally thought to be negligible in the magnetostrophic regime which leaves the Lorentz force as the only contribution in the first order force balance. Since this could lead to an awkward unbalanced acceleration Taylor (1963) concluded that the azimuthal Lorentz forces must largely cancel when integrated over the cylinders. In recognition of Taylor's work, the respective Lorentz force integral has been named the Taylor integral, the cancelation is referred to as Taylorization, and the dynamo configuration that is established by the cancelation is called a Taylor state.

Variations in the degree of Taylorization at an individual cylinder lead to an acceleration of this cylinder with respect to its neighbors. This shears $B_{s}$, the magnetic component perpendicular to the rotation axis (Taylor 1963), and thereby produces an azimuthal magnetic field. The Lorentz forces associated with this newly created field oppose the shear according 
to Lenz's law and therefore provide a restoring mechanism. The resulting oscillations are called torsional oscillations since the magnetic fieldlines effectively act as torsional springs in this process (Braginsky 1970). These oscillations travel as one-dimensional Alfvén waves in $s$ direction with a characteristic Alfvén speed that is proportional to the rms $B_{s}$ value over the geostrophic cylinders (Braginsky 1970).

Lorentz forces, gravitational forces, and viscous effects potentially couple the geostrophic cylinders to the inner core and the mantle. The coupled system can be approximated by an eigensystem that has been analyzed by several authors (Braginsky 1970; Buffett and Mound 2005; Mound and Buffett 2005; Dumberry and Mound 2008). The eigenperiods of this system are also proportional to the rms $B_{s}$ value and lie in the range of some years to decades for Earth (Braginsky 1970; Buffett and Mound 2005). Viscous effects, Ohmic effects, the coupling to the inner core, but in particular the coupling to the mantle can significantly damp torsional oscillations on time scales comparable to the oscillation period (Zatman and Bloxham 1997, 1999; Buffett and Mound 2005; Dumberry and Mound 2008). This could prevent the excitation of the individual standing eigenmodes that were envisioned by (Braginsky 1970) but would still allow for the onedimensional traveling Alfvén waves to survive for a limited time (Buffett and Mound 2005). We refer to Zatman and Bloxham (1999) for an extensive discussion of excitation and damping mechanisms.

The magnetostrophic balance, Taylorization, Taylor state, and torsional oscillations are intimately linked. Though the importance of torsional oscillations for the dynamo process itself may be minor, their presence can serve as an important indicator for the fact that viscous forces and inertial forces are indeed small in a planetary dynamo region. Moreover, torsional oscillations can provide estimates of the rms $B_{s}$ value and thus valuable information on the magnetic field inside the dynamo region which is not directly accessible by other observations. This highlights the potential importance of the work by Zatman and Bloxham (1997) and Bloxham (2002), who claim to have identified torsional oscillations of the predicted time scales in the geomagnetic field. In a recent study, however, Dumberry and Mound (2008) show that the damping due to the coupling to the mantle may actually be too strong for oscillations to be excited sufficiently. The decadal time scale, identified in core flow inversions and length-of-day variations, will then more likely reflect the dynamics of the excitation mechanism rather than the predicted eigenperiod (Dumberry and Mound 2008). Very likely, this excitation mechanism is the dynamo process which would thus manifest itself on much shorter time scales than the overturn time $t_{U}$.

Several authors have tried to identify torsional oscillations in dynamo simulations. Kuang (1999) and Dumberry and Bloxham (2003) show that viscous and inertial forces are still too influential in their Kuang and Bloxham model at $E=10^{-4}$. Busse and Simitev (2005) report to have found torsional oscillations at an Ekman number of $E=2 \times 10^{-5}$. Wicht and Christensen (2010) examine several dynamo models at different parameters and confirm that Taylorization and torsional oscillations go along with each other and can be observed when the viscous forces are small enough: No torsional oscillations can be identified at $\mathrm{E}=3 \times 10^{-4}$, first traces appear at $\mathrm{E}=3 \times 10^{-5}$, and the clearest signatures are present in their lowest Ekman number model at $\mathrm{E}=3 \times 10^{-6}$ referred to as E6 here. Figure 11 shows the zonal flow amplitude of geostrophic cylinders for a selected time span in model E6 where three waves are clearly discernable. The white lines mark the 'path' a wave would take when traveling with the Alfvén speed predicted for the propagation of torsional oscillations. The good agreement with the true propagation is an important test for the origin of these waves. The fact that the waves are rather short lived events demonstrates that the damping is large. While the strong damping of geomagnetic torsional oscillations is likely provided by the coupling to the mantle viscous effects may play this role in the numerical simulation. 
Fig. 11 Velocity of geostrophic cylinders in a selected time span of model E6. The white lines mark torsional oscillations that propagate with the predicted Alphvén velocity. The time is given in multiples of the magnetic diffusion time here

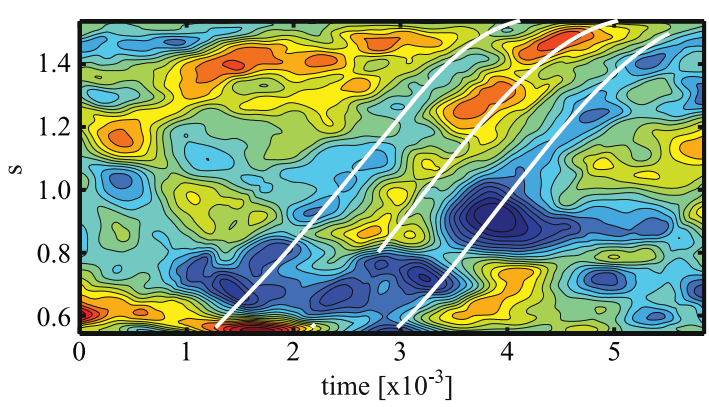

Wicht and Christensen (2010) also find that Reynolds stresses are too influential and prevent significant torsional oscillations as well as an efficient Taylorization at local Rossby number values around $\mathrm{Ro}_{\ell}=0.1$ where the simulations start to show reversals. Translated to Earth this would mean the identification of torsional oscillations in the outer core by Zatman and Bloxham (1997) and the value of $\mathrm{Ro}_{\ell}=0.09$ predicted by Christensen and Aubert (2006) are not compatible.

\section{Mantle Control}

Neither the longitudinal nor the equatorial symmetry are broken in the dynamo formalism developed in Sect. 3.1. The cmb field should therefore be perfectly axisymmetric and equatorially asymmetric when averaged over periods that are much longer than the typical time scale of magnetic field production, the convective overturn time of $t_{U} \approx 120 \mathrm{yr}$. There are, however, some indications that both these symmetries are broken in the geomagnetic field; we mainly discuss deviations from the axial symmetry in the following.

A particularly prominent persistent longitudinal feature are normal polarity flux patches in the radial magnetic field under Canada and Siberia with respective counterparts at comparable longitudes and latitudes south of the equator. They show up in paleomagnetic field models covering up to 5 Myr (Gubbins and Kelly 1993; Kelly and Gubbins 1997; Johnson and Constable 1995; Carlut and Courtillot 1998; Johnson et al. 2003) and can also be identified in archeomagnetic (Korte and Constable 2006) and historic (Jackson et al. 2000; Bloxham 2002) data (see Fig. 7).

These patches are reminiscent of the normal polarity patches close but outside the tangent cylinder in many dynamo simulations. However, as long is the longitudinal symmetry is not broken, these patches move around and also cease and emerge in an irregular fashion. Imposing a laterally varying $\mathrm{cmb}$ heat flux is a straight forward way to break both the axial and the equatorial symmetry. Seismic tomography data can be used to derive a geophysically motivated pattern by translating faster than average seismic velocities in the lowermost mantle ( $D^{\prime \prime}$-layer) into colder temperatures. Since the $\mathrm{cmb}$ is basically isothermal, colder temperatures yield a higher heat flux from the core to the mantle.

The procedure results in the typical 'tomographic' pattern shown in Fig. 12a of increased heat flux beneath the circum-Pacific rim which is often associated with the remains of colder subducted plates. Particularly high flux can be found beneath central America, eastern Siberia, and west of Australia (Masters et al. 2000). Aubert et al. (2008a) suggest that the relative amplitude of the lateral variation, $f^{\star}=\left(f_{\max }-f_{\min }\right) / 2 f_{0}$, may amount to about $f^{\star}=0.3$ for Earth. However, neither the mean heat flux $f_{o}$ nor the tomographic variation pattern are particularly well constrained. Earth's mean cmb heat flux may actually 

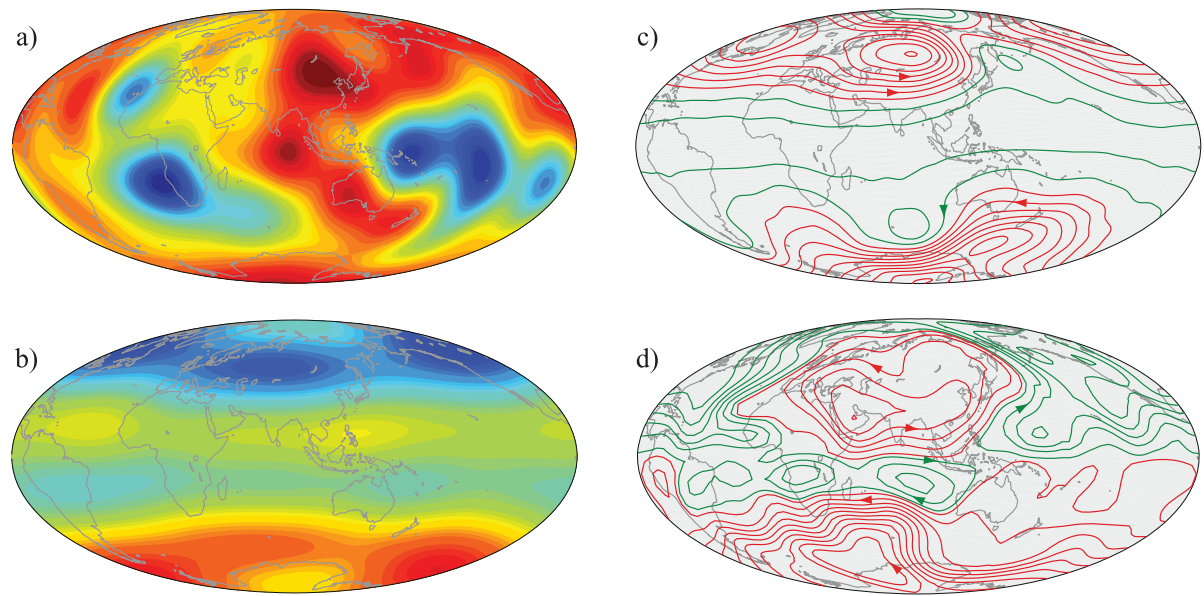

Fig. 12 (a) cmb heat flux pattern derived from a seismic tomography model for Earth's mantle (Masters et al. 2000), (b) time averaged radial magnetic field at the outer boundary in the dynamo model by Aubert et al. (2008a) that employs the pattern shown in panel (a) as the outer boundary condition, (c) associated time averaged horizontal flow underneath the viscous Ekman layer, (d) time averaged flow field (Amit and Olson 2006) based on a geomagnetic field model that covers the years 1840-1990 (Jackson et al. 2000)

be close enough to being adiabatic so that $f^{\star}$ can assume values larger than one and the heat flux would actually be subadiabatic in some regions. Several authors report that such a situation kills off the dynamo action in their numerical model (Glatzmaier et al. 1999; Olson and Christensen 2002; Willis et al. 2007) but this seems not to be the case in the simulations by Takahashi et al. (2008b).

The tomographic heat flux condition indeed promotes persistent magnetic flux patches at comparable locations as those found in the geomagnetic field (Olson and Christensen 2002; Christensen and Olson 2003; Gubbins et al. 2007; Aubert et al. 2008a). Figure 12b shows the results from Aubert et al. (2008a) which can be compared with the geomagnetic field model in Fig. 7. Figure 12 demonstrates that at least the northern hemispherical patches suggestively correlate with the two persistent flow cyclones that emerge in dynamo simulations employing tomographic thermal boundary condition. An associated down flow advectively collects the normal polarity field in a process similar to the one operating in the convective cyclones discussed in Sect. 5.1. The situation is less clear for the southern hemisphere.

Aubert et al. (2008a) averaged over a period equivalent to 0.7 Myr to yield this result. Olson and Christensen (2002) report that the magnetic field can be drastically different in snap shots and that the patches start to show up more clearly in their model when averaging over time spans equivalent to $2000 \mathrm{yr}$ or 17 convective turnover times. Is it a coincidence that these patches are so prominent in the historic geomagnetic field and seem to have moved little over the last 400 years (Jackson et al. 2000)? Willis et al. (2007) explore under which condition their dynamo model shows more persistent field morphologies that are quasi locked to the mantle. A not too small value of $f^{\star} \approx 0.3$ seems to be essential, but also a rather low Rayleigh number: $\mathrm{Ra}=1.5 \mathrm{Ra}_{c}$ at $\mathrm{E}=2.2 \times 10^{-4}$ in their case. This yields a dynamo with a relatively simple large scale solution that would never undergo field reversals. Also, the magnetic Reynolds number and thus the flow amplitude seem to be on the low side. Other authors use significantly more supercritical Rayleigh numbers (Olson and Christensen 2002; Aubert et al. 2008a). 
There is some hope that the averaging time required to recover the mantle influence in the fluid flow underneath Earth's cmb is shorter than for the magnetic field. The smaller length scale of the flow yields a shorter mixing time so that convective features are generally less persistent than magnetic structures (Huy et al. 2000). The underlying mantle control therefore has a better chance to reveal itself for shorter averaging periods. Aubert et al. (2007) explore this question with several dynamo simulations, calculating the correlation coefficients between averages of the flow at the top of the free stream over historic time spans with those over long time spans. Long refers to several hundred convective turnover times, while three overturn times, roughly $360 \mathrm{yr}$ for Earth, qualify as historic. This is about the time span for which geomagnetic models can provide an idea of the flow at the top of Earth's core. The correlation coefficient between the historic and the long averages reach encouraging values of 0.5 or higher, in particular for larger values of $f^{\star}$. This supports the conclusion by Amit and Olson (2006) that the geomagnetic flows models may already reveal a mantle signature.

The lateral $\mathrm{cmb}$ heat flux variations alone would drive a pure thermal winds system. Aubert et al. (2007) demonstrate that the time averaged horizontal flows in their simulations are still dominated by thermal winds even though the models involve a strong component of core convection. However, the effects of deeper convection mix with the boundary induced features in a complex way (Olson and Christensen 2002; Amit and Olson 2006; Aubert et al. 2007). The latter are modified and possibly shifted westward with respect to the thermal mantle structure, and in some regions the deeper core convection seem to dominate altogether. This is particularly true for the zonal flows inside and close to the tangent cylinder (Amit and Olson 2006) that we discussed in Sect. 5.4. Amit and Olson (2006) remark that the time averaged flow fields show a significant equatorial asymmetry, those based on the historic geomagnetic field (Fig. 12) as well as the long-time averages from dynamo simulations that use the topographic boundary conditions. At least in the latter case, this must be due to the cmb heat flux pattern.

How deep do the persistent flow structures reach into the core? The equatorial asymmetry implies that the flow cyclones that have been identified underneath the cmb can not be the straight forward manifestation of convective cyclones that go all the way through the outer core. Aubert et al. (2008a) suggest that at least the cyclones beneath Asia may reach down to the inner core and increases the removal of heat and light elements from it's eastern hemisphere. This could help to explain why seismic waves that pass through the top hundred $100 \mathrm{~km}$ of this hemisphere are faster, more isotropic, and more attenuated (Tanaka and Hamaguchi 1997; Niu and Wen 2001; Yu and Wen 2006). Laboratory experiments have shown that the inner-core growth rate influences the structure of the hexagonal-closed-pack (hcp) inner-core iron: slower iron solidification favors wider spaced and more oriented dendritic growth (Bergman 2003). The lower density yields a slower seismic velocities and the larger degree of orientation yields a higher seismic anisotropy since hcp iron crystals have a seismically faster axis. A faster solidification of the inner core's eastern hemisphere, promoted by the more efficient removal of heat and light elements, could therefore explain the variation in the seismic signal by causing a denser and more random crystal structure in this region.

Freezing the outer $100 \mathrm{~km}$ of Earth's inner core would take 100-300 Myr when assuming typical growth rates of $0.3-0.9 \mathrm{~mm} / \mathrm{yr}$ (Labrosse et al. 2001). It seems not implausible that the larger scale $\mathrm{cmb}$ heat flux pattern remained more or less unaltered during such a time span since mantle structures typically change on time scales of some $10 \mathrm{Myr}$ to $100 \mathrm{Myr}$. Gravitational forces between inner core and mantle could provide the necessary locking between the inner core and the persistent cyclone (Aubert et al. 2008a). 
The potential mantle control of the dynamo mechanism is also interesting in the context of magnetic field reversals. Glatzmaier et al. (1999) and Kutzner and Christensen (2004) show that the $\mathrm{cmb}$ heat flux pattern can influence the reversal rate in a numerical dynamo model. That this effect may indeed play a role in the geomagnetic field is supported by the fact that Earth's reversal rate seems to change over the time scales of mantle convection (Loper 1992). Besides comparing cases with homogeneous and tomographic $\mathrm{cmb}$ heat flux Glatzmaier et al. (1999) as well as Kutzner and Christensen (2004) also impose other patterns to explore their influence. Kutzner and Christensen (2004) find that reversals are promoted when the heat flux in the equatorial region in increased while the heat flux in the polar regions seems to have no influence on the reversal rate. The higher equatorial heat flux increases the convective flow vigor outside the tangent cylinder. It may thus simply yield larger $\mathrm{Ro}_{\ell}$ values and thereby increase the reversals likelihood. Glatzmaier et al. (1999), however, come to an opposing conclusion. In their model the heat flux in the polar regions is more influential: An increase promotes stronger convective plumes inside the tangent cylinder. The associated stronger thermal winds increase the toroidal fields that are created at the boundary to the tangent cylinder. These stronger toroidal fields in turn yield stronger poloidal fields in general and a stronger dipole field in particular which is less prone to reversals (Glatzmaier and Roberts 1997). The toroidal and poloidal field production inside and close to the tangent cylinder plays little role in the standard model employed by Kutzner and Christensen (2004).

Some paleomagnetic studies also claim that during field reversals the magnetic pole preferentially follows two longitude bands which are about $180^{\circ}$ apart and roughly coincide with the areas of increased cmb heat flow in the tomographic models (Laj et al. 1991; Merrill and McFadden 1999). We have discussed in Sect. 6 that the dipole field decreases during a reversal and subsequently recovers with opposite polarity. The transitional pole positions therefore reflect virtual geomagnetic poles (VGP) rather than true dipole poles and depend on the site the paleomagnetic data originate from. Coe et al. (2000) and Kutzner and Christensen (2004) show that the preferred bands can largely be recovered in a numerical dynamo model when imposing a tomographic $\mathrm{cmb}$ heat flux. The increased flux underneath the Pacific rim drives somewhat stronger convective up and down streams in these regions which in turn promote the appearance of more intense magnetic flux patches of both polarities. These patches may dominate the magnetic field during transitional times sufficiently enough to largely determine the VGPs for paleomagnetic sites in their respective sphere of influence.

\section{Tidally Driven and Precession Driven Dynamos}

Bullard (1949) apparently was the first to consider tidal deformation of the core mantle boundary and precession of the rotation axis as possible energy sources for the geodynamo. Tides never looked like a plausible driving agent in the Earth, but it cannot be ruled out that precession is driving the geodynamo. In this section, we look at how precession or tides can in principle drive a dynamo and whether the parameters of the planets in the solar system are such that any of these effects is likely to occur.

Let us first assume that a planetary core is adiabatically stratified so that we do not need to worry about buoyancy forces. It helps to next take the point of view of a fluid dynamicist and to consider tidal deformation or precession rate as a control parameter which can be increased at will starting from zero. As long as the control parameter is small, the excited flow is laminar. Tides and precession can conveniently be discussed together because the 
Fig. 13 Streamlines for laminar flow driven by tides or precession. The axis labels are for tidal flow. For the case of precession, there is no tidal body, the oblique axis should be labeled "rotation axis of the fluid", and the rotation axis of the mantle is vertical

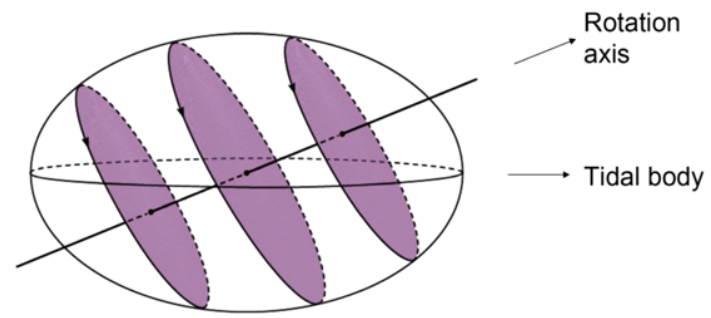

laminar flows are similar in both cases. Let us first look at tides: A planet rotates about an axis which is also an axis of symmetry. The liquid core of the planet, viewed from an inertial frame of reference, rotates about the same axis at the same rate. Fluid particles move on circular paths. If one now adds tidal deformation to the picture, the rotation axis is no longer an axis of symmetry and fluid particles circulate on elliptical streamlines (see Fig. 13). It is also noteworthy that the rotation axis is not joining the centers of the ellipses.

Let us now turn to precession. At zero precession rate, core and mantle rotate again about the same axis. If the mantle starts to precess, the core will have to follow. In a steady state, the rotation axes of core and mantle may differ, but they have to precess at the same rate about the same precession axis. One then arrives at the same streamline pattern as in Fig. 13, except that the rotation axis of the planet is now vertical in the figure and still is a symmetry axis of the planet. In a gas planet, the same figure applies, with the "core mantle boundary" replaced by a "surface of constant pressure".

Known antidynamo theorems nearly exclude that the flow in Fig. 13 is a dynamo. Streamlines in Fig. 13 lie in planes. There is an antidynamo theorem stating that dynamo action in that case is impossible provided that conductor-vacuum interfaces are also parallel to those planes, which is not the case in Fig. 13. Even though dynamo action is not excluded by antidynamo theorems, it seems unlikely that the flow of Fig. 13 is a dynamo at magnetic Reynolds numbers of planetary relevance, if at all.

Something needs to happen to the laminar precessional or tidal flow before it becomes interesting for the dynamo effect. If one increases the control parameter, instabilities will occur. Instabilities lead to a more complicated, three dimensional flow potentially capable of dynamo action. Our knowledge about instabilities is more complete for precession than for tides. Instabilities are best studied close to their onset, i.e. in a narrow range of control parameters, which are easier to fine tune in numerical simulations than in experiments. A sphere is a much more convenient geometry for numerics than an ellipsoid. Tidal effects cannot be dissociated from ellipsoidal boundaries, whereas precessing spheres and ellipsoids produce similar instabilities.

Three types of instabilities are known from precessing spheres (Lorenzani and Tilgner 2001). The first occurs in the boundary layers and stays confined to them, so that it has little effect on the flow globally. The second type occurs in internal shear layers. It is not understood why these internal shear layers appear on top of the flow in Fig. 13 except for the strongest of them (Busse 1968), but they are a robust feature of both experiments and simulations. The third type is a consequence of the streamline geometry in Fig. 13: Streamlines are elliptical, giving rise to the so called "elliptical instability" (Kerswell 2002), and there is differential motion between adjacent planes in which the ellipses lie, giving rise to shear instabilities. The third type is by far the most accessible to theoretical analysis and is understood best. However, early experiments conveyed the intuition that the second type is the most important (Malkus 1968; Vanyo et al. 1995). Clearly, different instabilities set in 
Fig. 14 Sketch of the structure of unstable precession driven flow (from Lorenzani and Tilgner 2001)

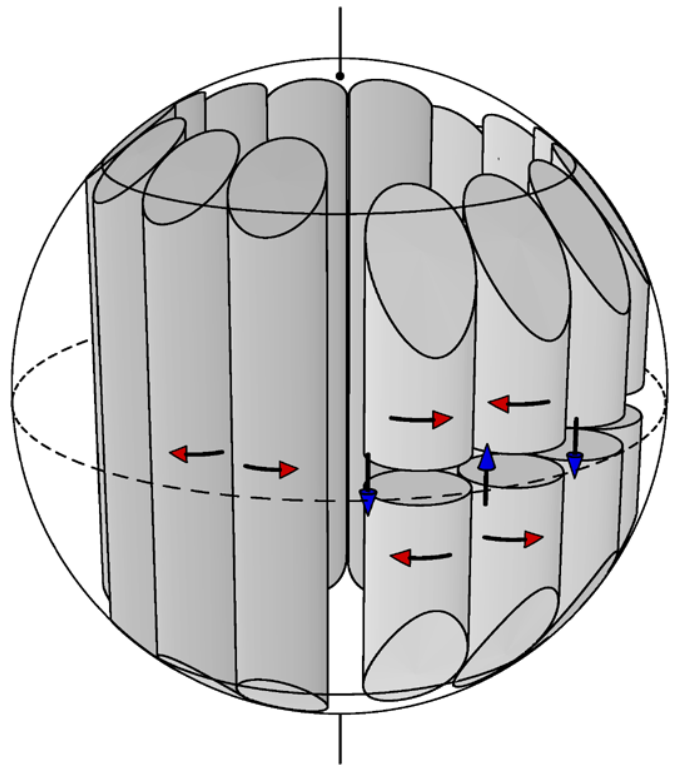

first depending on the control parameters and we do not know at present what to expect for planetary values.

Figure 14 is a sketch of the elliptical instability as it was observed in precession driven flow. Similar to convection, columnar vortices drifting around the rotation axis are excited. In convection, instability starts from an axisymmetric ground state, but it starts from a nonaxisymmetric flow in the precessional or tidal case. For that reason, convection excites one set of columns or one wave, whereas precession or tides excite two of them. In the schematic Fig. 14, the azimuthal wave number of the two sets of vortices differs by 1 (it would have to differ by 2 in the instability of an equatorial tide). One of the two sets has qualitatively the same structure (and in particular the same helicity distribution) as thermal convection.

The elliptical instability also occurs in the simplest tidal flow inside a planet, caused by a moon in the equatorial plane. We know for this geometry a rigorous upper bound for the growth rate of elliptical instabilities in inviscid fluids (Kerswell 2002). This upper bound is proportional to the ellipticity of the streamlines if the tidal deformation is small. We can thus proceed as follows to determine whether an elliptical instability is likely in any given planet: From the masses of planet and moon, $M_{\text {Planet }}$ and $M_{\text {Moon }}$, the radius of the planet $R_{\text {Planet }}$, and the distance from the planet to the moon, $R_{\text {Moon }}$, one computes the amplitude of the equilibrium tide $h_{T}$ at the outer edge of the conducting region of the planet which has radius $R_{c}$. In a planet of uniform density, which is assumed in this simple estimate, the ellipticity of the tidal deformation is independent of depth and one finds:

$$
\frac{h_{T}}{R_{c}}=\frac{1}{2} \frac{M_{\text {Moon }}}{M_{\text {Planet }}}\left(\frac{R_{\text {Planet }}}{R_{\text {Moon }}}\right)^{3}
$$

$h_{T} / R_{c}$ is listed in Table 2 and gives (apart form a prefactor of order 1 ) the upper bound of the growth rate in multiples of $1 / \Omega$, where $\Omega$ is the angular frequency of rotation of the planet. Exact growth rates of elliptical instabilities tend to be of the same order as this upper bound, so that we go on with $h_{T} / R_{c}$ as a representative value for the growth rate. This growth needs to overcome viscous dissipation. In a flow with Ekman number $\mathrm{E}$, defined as $\mathrm{E}=v /\left(\Omega R_{c}^{2}\right)$, 
Table 2 Some planetary parameters as defined and used in the text

\begin{tabular}{lllllll}
\hline Planet & Moon & $R_{c}[\mathrm{~m}]$ & $M_{\text {Planet }}[\mathrm{kg}]$ & $R_{\text {Moon }}[\mathrm{m}]$ & $M_{\text {Moon }}[\mathrm{kg}]$ & $h_{T} / R_{C}$ \\
\hline Earth & Moon & $3.47 \times 10^{6}$ & $5.97 \times 10^{24}$ & $3.84 \times 10^{8}$ & $7.35 \times 10^{22}$ & $2.81 \times 10^{-8}$ \\
Jupiter & Io & $5.71 \times 10^{7}$ & $1.90 \times 10^{27}$ & $4.22 \times 10^{8}$ & $8.91 \times 10^{22}$ & $1.59 \times 10^{-7}$ \\
Saturn & Titan & $2.53 \times 10^{7}$ & $5.68 \times 10^{26}$ & $1.22 \times 10^{9}$ & $1.35 \times 10^{23}$ & $1.43 \times 10^{-8}$ \\
Uranus & Ariel & $2.05 \times 10^{7}$ & $8.70 \times 10^{25}$ & $1.91 \times 10^{8}$ & $1.44 \times 10^{21}$ & $1.94 \times 10^{-8}$ \\
Neptune & Triton & $1.98 \times 10^{7}$ & $1.03 \times 10^{26}$ & $3.55 \times 10^{8}$ & $3.40 \times 10^{23}$ & $5.31 \times 10^{-7}$ \\
\hline
\end{tabular}

$v$ being the kinematic viscosity, the dissipation rate is on the order of $E^{1 / 2}$ in the presence of no slip boundaries and on the order of $\mathrm{E}$ for free slip boundaries. Commonly accepted values for the Earth's core are $\mathrm{E}=10^{-14}-10^{-15}$. It is more shaky to assign an Ekman number to a gas planet because $v$ is depth dependent, but $\mathrm{E}$ around $10^{-20}$ is a reasonable value. At any rate, an elliptical instability is likely in all gas planets, but unlikely inside the Earth. Coexistence with convective flows does not change the argument because of a separation of time scales (Tilgner 2007). It is more difficult to take a turbulent viscosity into account with any confidence (Fabijonas and Holm 2003).

A more elaborate calculation is required for precession. Precession is most important in planets with a big moon well outside the equatorial plane. This singles out the Earth (in which precessional instability is plausible but whether it actually happens depends on the exact value of E) and the Neptune/Triton pair (in which precessional instability is likely).

All these considerations were about the onset of instability. Once the new flow has grown, it will interact with convection if it is present. The mutual influence of both flows has not been studied yet. On the other hand, it is known that precession driven unstable flow can maintain a magnetic field (Tilgner 2005).

\section{Conclusion}

Three dimensional self-consistent numerical dynamo simulations are capable of reproducing many features of the geomagnetic field. Some of its basic properties are fairly robust and can be found over a large range of parameters. A dominant axial dipole component, strong flux patches at latitudes close to where the tangent cylinder touches the outer boundary, and weaker or inverse field inside the tangent cylinder can already be found in rather simple large scale models with Ekman numbers of order $\mathrm{E}=10^{-3}$. If the Rayleigh number is chosen high enough to yield a sufficiently strong inertial force at a local Rossby number around $\mathrm{Ro}_{\ell}=0.1$, these dynamos also show Earth-like reversals.

Though Ekman number and magnetic Prandtl number are far away from realistic values even simple models seem able to capture the fundamental dynamics correctly. In order to translate the dimensionless simulations to absolute physical values the results have to be extrapolated to realistic parameters. The simplicity of the scaling laws that have been suggested for the extrapolation (Christensen and Aubert 2006; Amit and Olson 2008; Takahashi et al. 2008a; Christensen et al. 2009; Christensen [doi:10.1007/s11214-009-9553-2], this issue) support the view that the numerical simulations, planetary dynamos, and even dynamos of fast rotating stars share the same fundamental dynamo processes. The fact that the small scale flow dynamics is certainly not captured correctly seems to play a secondary role. Even the simple larger Ekman number dynamos can thus prove useful, for example for unraveling the fundamental dynamo action and for understanding and predicting dipole behavior including magnetic field reversals. 
However, our analysis of the Ekman number dependence reveals that some detail features may indeed be more Earth like at lower Ekman numbers. The low latitude magnetic field at the outer boundary may become more similar to the historic magnetic field for $\mathrm{E} \leq 3 \times 10^{-5}$. More studies are needed to understand this issue and to explore the parameter dependence. Also, torsional oscillations, which may form an important part of the decadal geomagnetic field variations, only start to appear in simulations with $\mathrm{E} \leq 3 \times 10^{-5}$. And even for $\mathrm{E}=$ $3 \times 10^{-6}$ they travel much slower than in Earth (Wicht et al. 2009). While these aspects seem secondary as far as the fundamental dynamo process is concerned, they may prove essential for modeling and explaining Earth's smaller scale field structure and its evolution on time scales of decades to centuries.

A recent simulations by Kageyama et al. (2008) at $\mathrm{E}=10^{-6}$ indicates a more drastic regime change: Sheetlike convective columns are restricted to the region closer to the tangent cylinder and the magnetic field is rather small scale and non-dipolar. However, the results are inconclusive since the magnetic field is still developing and may only be transient.

The influence of the magnetic Prandtl number has not been explored very extensively so far owed to the fact that lower magnetic Prandtl numbers can only be assumed at low Ekman numbers. Unfortunately, low E and low Pm values both increase the numerical costs of the computations. Pm provides a measure for the ratio between the small length scales in flow and magnetic field, respectively. The low Pm value around $10^{-6}$ in planetary dynamo regions mean that the magnetic field is insensitive to the small scale turbulent flow structures. Numerical dynamo simulations have not ventured below $\mathrm{Pm}=0.1$ and have thus not really explored the effects of this scale separation (Schaeffer and Cardin 2006).

For a less generic prediction of the evolution of individual features in Earth's magnetic field the additional problem remains that the outer boundary field of a numerical simulations generally never exactly matches the geomagnetic field. Data assimilations techniques can help here. Kuang et al. (2009) demonstrate that imposing the history magnetic field from 1900 to 2000 in $20 \mathrm{yr}$ intervals to a dynamo simulation can reduce the forecast error by $30 \%$. They show that the data assimilation technique gradually changes the dynamic state of the outer core and that its influence reaches progressively deeper into the core over time. The use of more realistic thermal boundary conditions imposed by Earth's mantle is another attempt to make the numerical simulations more realistic. Whether this can already have an effect on the historic time scale of decades to centuries remains dubious but the long time scale influence certainly yields intriguing results.

The coming years will probably see dynamo simulations at Ekman numbers of $10^{-7}$ and magnetic Prandtl numbers of order $10^{-2}$. Naturally, this will largely depend on the computing power available for dynamo simulations. The better scalability of the newer local numerical methods (Wicht et al. 2009) may help to reach this goal on massively parallel computing systems. The disadvantage of these ambitious simulations is that the small scale solutions are difficult to interpret and that the runs cover comparatively short periods. This will make a detailed analysis of the internal dynamics impossible, likely preclude the simulation of reversals, and rule out an exploration of the long-term mantel control.

Not too long ago, convection driven geodynamo simulations where deemed realistic when they produced a dipole dominated magnetic field. The fact that they can also undergo reversals was seen as a final prove for their validity (Glatzmaier and Roberts 1995b). Since then, the models have been refined to replicate more and more features of the geomagnetic field and to provide an explanation for the observations. Several spherical selfconsistent dynamo codes have been developed over the last years and some have been made freely available to other researchers. At least the models with larger Ekman numbers around $\mathrm{E}=10^{-3}$ already run sufficiently fast on a multi-core PC so that the numerical costs also no longer pose a barrier. Does this leave room for the highly idealized and 
parameterized models that were so common in the past: kinematic dynamos where simple flow fields are prescribed or mean field approaches where the small scale dynamo action is parameterized all together? These models may still remain useful where really fundamental questions are addressed or the numerical limitations still prevent the use of the self-consistent full approaches. One example is the statistical exploration of the reversal likelihood. The maximum number of reversals found in a self-consistent full model is about 160 (Wicht et al. 2009). Parameterized models, however, can afford to simulate thousands of reversals and therefore offer a much better statistics (Ryan and Sarson 2007; Schmitt et al. 2001). In addition, they allow to vary the parameters to explore which combination provides the most Earth-like statistics. Another example is the possible coupling of the magnetospheric and the internal dynamo at Mercury which has so far not been explored in a self-consistent full model. The simple parameterized approach by Glassmeier et al. (2007) suggests that this may indeed yield interesting results and explain the planet's low magnetic field strength. However, the results of the parameterized models should always be interpreted with care and should be reconciled with full models as far as possible.

Self-consistent full numerical dynamo models are increasingly successful in explaining the magnetic field of the other planets in our solar system. Variations of the inner-core size, the driving mode, and the outer thermal boundary condition seem to play key roles here. We refer the interested reader to Stanley and Glatzmaier [doi:10.1007/s11214-009-9573-y], this issue). Tidally and precession driven dynamos offer an interesting alternative, but the respective numerical models have a long way to go to catch up with convection driven dynamo simulations.

\section{References}

H. Amit, P. Olson, Phys. Earth Planet. Inter. 155, 120 (2006)

H. Amit, P. Olson, Phys. Earth Planet. Inter. 166, 226 (2008)

A.P. Anufriev, C.A. Jones, A.M. Soward, Phys. Earth Planet. Inter. 152, 163 (2005)

M. Ardes, F. Busse, J. Wicht, Phys. Earth Planet. Inter. 99, 55 (1996)

J. Aubert, J. Fluid Mech. 542, 53 (2005)

J. Aubert, H. Amit, G. Hulot, Phys. Earth Planet. Inter. 160, 143 (2007)

J. Aubert, H. Amit, G. Hulot, P. Olson, Nature 454, 758 (2008a)

J. Aubert, J. Aurnou, J. Wicht, Geophys. J. Int. 172, 945 (2008b)

J. Aubert, S. Labrosse, C. Poitou (2009). doi:10.1111/j.1365-246X.2009.04361.x

J. Aubert, J. Wicht, Earth. Planet. Sci. Lett. 221, 409 (2004)

J. Aurnou, S. Andreadis, L. Zhu, P. Olson, Earth Planet. Sci. Lett. 212, 119 (2003)

M. Bergman, J. Cryst. Growth 255, 204 (2003)

J. Bloxham, Geophys. Res. Lett. 39 (2002). doi:10.1029/2001GL014543

J. Bloxham, S. Zatman, M. Dumberry, Nature 420, 65 (2002)

S. Braginsky, Geomag. Aeron. 10, 1 (1970)

S. Braginsky, P. Roberts, Geophys. Astrophys. Fluid Dyn. 79, 1 (1995)

B.A. Buffett, J.E. Mound, J. Geophys. Res. 110, B08104 (2005)

E. Bullard, Proc. R. Soc. A 197, 433 (1949)

F. Busse, J. Fluid Mech. 33, 739 (1968)

F. Busse, J. Fluid Mech. 44, 441 (1970)

F.H. Busse, Geophys. Res. Lett. 29, 070000 (2002)

F.H. Busse, R. Simitev, in Fluid Dynamics and Dynamos in Astrophysics and Geophysics, ed. by A.M. Soward, C.A. Jones, D.W. Hughes, N.O. Weiss, (2005), p. 359

F.H. Busse, R.D. Simitev, Geophys. Astrophys. Fluid Dyn. 100, 341 (2006)

F.H. Busse, R.D. Simitev, Phys. Earth Planet. Inter. 168, 237 (2008)

J. Carlut, V. Courtillot, Geophys. J. Int. 134, 527 (1998)

U. Christensen, J. Aubert, Geophys. J. Int. 116, 97 (2006)

U. Christensen, P. Olson, Phys. Earth Planet. Inter. 138, 39 (2003) 
U. Christensen, J. Wicht, in Treatise on Geophysics, ed. by P. Olson. Core Dynamics, vol. 8 (Elsevier, Amsterdam, 2007), p. 245

U.R. Christensen, J. Fluid Mech. 470, 115 (2002)

U.R. Christensen, Nature 444, 1056 (2006)

U.R. Christensen, J. Aubert, F.H. Busse et al., Phys. Earth Planet. Inter. 128, 25 (2001)

U.R. Christensen, V. Holzwarth, A. Reiners, Nature 457, 167 (2009)

R. Coe, L. Hongre, A. Glatzmaier, Philos. Trans. R. Soc. Lond. A 358, 1141 (2000)

R.S. Coe, G.A. Glatzmaier, Geophys. Res. Lett. 33, 21311 (2006)

P. Davidson, An Introduction to Magnetohydrodynamics (Cambridge University Press, Cambridge, 2001)

P. Driscoll, P. Olson, Earth Planet. Sci. Lett. 282, 24 (2009)

M. Dumberry, J. Bloxham, Phys. Earth Planet. Inter. 140, 29 (2003)

M. Dumberry, J.E. Mound, J. Geophys. Res. 113, B03102 (2008)

M. Evonuk, Astrophys. J. 673, 1154 (2008)

C. Eymin, G. Hulot, Phys. Earth Planet. Inter. 152, 200 (2005)

B. Fabijonas, D. Holm, Phys. Rev. Lett. 90, 124501 (2003)

K. Glassmeier, H. Auster, U. Motschmann, Geophys. Res. Lett. 34, 22201 (2007)

G. Glatzmaier, J. Comput. Phys. 55, 461 (1984)

G. Glatzmaier, Annu. Rev. Earth Planet. Sci. 30, 237 (2002)

G. Glatzmaier, R. Coe, in Treatise on Geophysics, ed. by P. Olsen. Core Dynamics, vol. 8 (Elsevier, Amsterdam, 2007), p. 283

G. Glatzmaier, R. Coe, L. Hongre, P. Roberts, Nature 401, 885 (1999)

G. Glatzmaier, P. Roberts, Phys. Earth Planet. Inter. 91, 63 (1995a)

G. Glatzmaier, P. Roberts, Nature 337, 203 (1995b)

G. Glatzmaier, P. Roberts, Physica D 97, 81 (1996a)

G. Glatzmaier, P. Roberts, Science 274, 1887 (1996b)

G. Glatzmaier, P. Roberts, Contemp. Phys. 38, 269 (1997)

E. Grote, F. Busse, Phys. Rev. 62, 4457 (2000)

E. Grote, F. Busse, Fluid Dyn. Res. 28, 349 (2001)

E. Grote, F. Busse, A. Tilgner, Phys. Rev. E 60, 5025 (1999)

E. Grote, F. Busse, A. Tilgner, Phys. Earth Planet. Inter. 117, 259 (2000)

D. Gubbins, Phys. Earth Planet. Inter. 128, 3 (2001)

D. Gubbins, P. Kelly, Nature 365, 829 (1993)

D. Gubbins, A.P. Willis, B. Sreenivasan, Phys. Earth Planet. Inter. 162, 256 (2007)

H. Harder, U. Hansen, Geophys. J. Int. 161, 522 (2005)

M. Heimpel, J. Aurnou, J. Wicht, Nature 438, 193 (2005)

M.L. Huy, M. Mandea, L.L. Jean-Mouël, A. Pais, Earth Planets Space 52, 163 (2000)

A. Jackson, Phys. Earth Planet. Inter. 103, 293 (1997)

A. Jackson, Nature 424, 760 (2003)

A. Jackson, J. Bloxham, D. Gubbins, in Dynamics of Earth's Deep Interior and Earth Rotation (1993), p. 97

A. Jackson, C. Finlay, in Treatise on Geophysics, ed. by M. Kono. Geomagnetism, vol. 5 (Elsevier, Amsterdam, 2007), p. 147

A. Jackson, A. Jonkers, M. Walker, Philos. Trans. R. Soc. Lond. A 358, 957 (2000)

D. Jault, in Earth's Core and Lower Mantle, ed. by C.A. Jones, A.M. Soward, K. Zhang (2003), p. 56

D. Jault, C. Gire, J.-L. LeMouël, Nature 333, 353 (1988)

D. Jault, J.-L. LeMouël, Geophys. Astrophys. Fluid Dyn. 48, 273 (1989)

C. Johnson, C. Constable, Geophys. J. Int. 122, 489 (1995)

C. Johnson, C. Constable, L. Tauxe, Science 300, 2044 (2003)

C. Jones, Philos. Trans. R. Soc. Lond. A 358, 873 (2000)

C. Jones, in Treatise on Geophysics, ed. by P. Olson. Core Dynamics, vol. 8 (Elsevier, Amsterdam, 2007), p. 131

C. Jones, P. Roberts, J. Fluid Mech. 404, 311 (2000)

A. Kageyama, T. Miyagoshi, T. Sato, Nature 454, 1106 (2008)

A. Kageyama, M. Ochi, T. Sato, Phys. Rev. Lett. 82, 5409 (1999)

A. Kageyama, T. Sato, Phys. Plasmas 2, 1421 (1995)

A. Kageyama, T. Sato, Plasma Phys. Contr. Fusion 39, 83 (1997)

A. Kageyama, M. Yoshida, J. Phys. Conf. Ser. 16, 325 (2005)

P. Kelly, D. Gubbins, Geophys. J. Int. 128, 315 (1997)

R. Kerswell, Annu. Rev. Fluid Mech. 34, 83 (2002)

M. Kono, P. Roberts, Rev. Geophys. 40, 1013 (2002). doi:10.1029/2000RG000102

M. Korte, C. Constable, Geophys. J. Int. (2006). doi:10.1111/j.1365-246X.2006.03088.x

W. Kuang, Phys. Earth Planet. Inter. 116, 65 (1999) 
W. Kuang, J. Bloxham, Nature 389, 371 (1997)

W. Kuang, J. Bloxham, J. Comput. Phys. 153, 51 (1999)

W. Kuang, A. Tangborn, Z. Wei, T. Sabaka, Geophys. J. Int. 179, 1458 (2009)

C. Kutzner, U. Christensen, Geophys. Res. Lett. 27, 29 (2000)

C. Kutzner, U. Christensen, Phys. Earth Planet. Inter. 131, 29 (2002)

C. Kutzner, U. Christensen, Geophys. J. Int. 157, 1105 (2004)

S. Labrosse, Phys. Earth Planet. Inter. 140, 127 (2003)

S. Labrosse, J.-P. Poirier, J.-L. Le Mouël, Earth Planet. Sci. Lett. 190, 111 (2001)

C. Laj, A. Mazaud, R. Weeks, Nature 351, 447 (1991)

R. Leonhardt, K. Fabian, Earth Planet. Sci. Lett. 253, 172 (2007)

J. Li, T. Sato, A. Kageyama, Science 295, 1887 (2002)

J. Lister, B. Buffett, Phys. Earth Planet. Inter. 91, 17 (1995)

J. Liu, P.M. Goldreich, D.J. Stevenson, Icarus 196, 653 (2008)

D.E. Loper, Geophys. Res. Lett. 19, 25 (1992)

S. Lorenzani, A. Tilgner, J. Fluid Mech. 447, 111 (2001)

V. Malkus, Science 160, 259 (1968)

G. Masters, G. Laske, H. Bolton, A. Dziewonski, in AGU Monograph, ed. by S. Karato, F.A.R. Lieberman, G. Masters, L. Stixrude. Earth's Deep Interior: Mineral Physics and Tomography from the Atomic to the Global Scale, vol. 117 (American Geophysical Union, Washington, 2000), p. 63

H. Matsui, H. Okuda, Int. J. Comput. Fluid Dyn. 19, 15 (2005)

S. Maus, M. Rother, C. Stolle et al., Geochem. Geophys. Geosyst. 7, 7008 (2006)

D.G. McMillan, G.R. Sarson, 2003, AGU Fall Meeting Abstracts, C280+

R. Merrill, P. McFadden, Rev. Geophys. 37, 201 (1999)

H. Moffat, Magnetic Field Generation in Electrically Conducting Fluids (Cambridge University Press, Cambridge, 1978)

J.E. Mound, B.A. Buffett, J. Geophys. Res. 110, B08103 (2005)

F. Nimmo, in Treatise on Geophysics, ed. by P. Olson. Core Dynamics, vol. 8 (Elsevier, Amsterdam, 2007), p. 31

N. Nishikawa, K. Kusano, Phys. Plas. 15, 082903 (2008)

F. Niu, L. Wen, Nature 410, 1081 (2001)

M.M. Ochi, A. Kageyama, T. Sato, Phys. Plas. 6, 777 (1999)

D.E. Ogden, G.A. Glatzmaier, R.S. Coe, Geophys. Astrophys. Fluid Dyn. 100, 107 (2006)

P. Olson, U. Christensen, Geophys. J. Int. 151, 809 (2002)

P. Olson, U. Christensen, Earth Planet. Sci. Lett. 250, 561 (2006)

P. Olson, U. Christensen, G. Glatzmaier, J. Geophys. Res. 104, 10,383 (1999)

E. Parker, Astrophys. J. 121, 293 (1955)

G. Roberts, Philos. Trans. R. Soc. A 271, 411 (1972)

P. Roberts, Philos. Trans. R. Soc. Lond., A 263, 93 (1968)

P. Roberts, in Irreversible Phenomena and Dynamical Systems Analysis in Geosciences, ed. by C. Nicolis, G. Nicolis (Reidel, Dordrecht, 1987), p. 73

P. Roberts, G. Glatzmaier, Geophys. Astrophys. Fluid Dyn. 94, 47 (2001)

J. Rotvig, Phys. Earth Planet. Inter. 176, 69 (2009)

J. Rotvig, C. Jones, Phys. Rev. E 66, 056308 (2002)

D.A. Ryan, G.R. Sarson, Geophys. Res. Lett. 34, 2307 (2007)

N. Schaeffer, P. Cardin, Earth Planet. Sci. Lett. 245, 595 (2006)

D. Schmitt, M.A.J.H. Ossendrijver, P. Hoyng, Phys. Earth Planet. Inter. 125, 119 (2001)

R. Simitev, F. Busse, J. Fluid Mech. 532, 365 (2005)

R.D. Simitev, F.H. Busse, Europhys. Lett. 85, 19001 (2009)

B. Sreenivasan, C.A. Jones, Geophys. Res. Lett. 32, L20301 (2005)

B. Sreenivasan, C.A. Jones, Geophys. Astrophys. Fluid Dyn. 100, 319 (2006)

S. Stanley, J. Bloxham, Nature 428, 151 (2004)

S. Stanley, J. Bloxham, Icarus 184, 556 (2006)

S. Stanley, J. Bloxham, W. Hutchison, M. Zuber, Earth Planet. Sci. Lett. 234, 341 (2005)

S. Stanley, M.T. Zuber, J. Bloxham, Geophys. Res. Lett. 34, 19205 (2007)

S. Stellmach, U. Hansen, Phys. Rev. E 70, 056312 (2004)

F. Takahashi, M. Matsushima, Geophys. Res. Lett. 33, L10202 (2006)

F. Takahashi, M. Matsushima, Y. Honkura, Science 309, 459 (2005)

F. Takahashi, M. Matsushima, Y. Honkura, Earth Planets Space 59, 665 (2007)

F. Takahashi, M. Matsushima, Y. Honkura, Phys. Earth Planet. Inter. 167, 168 (2008a)

F. Takahashi, H. Tsunakawa, M. Matsushima, N. Mochizuki, Y. Honkura, Earth Planet. Sci. Lett. 272, 738 (2008b) 
S. Tanaka, H. Hamaguchi, J. Geophys. Res. 102, 2925 (1997)

J. Taylor, Proc. R. Soc. Lond. A 274, 274 (1963)

A. Tilgner, Geophys. Astrophys. Fluid Dyn. 98, 225 (2004)

A. Tilgner, Phys. Fluids 17, 034104 (2005)

A. Tilgner, in Treatise on Geophysics, ed. by G. Schubert (Elsevier, Amsterdam, 2007), p. 207

A. Tilgner, F.H. Busse, J. Fluid Mech. 332, 359 (1997)

J. Vanyo, P. Wilde, P. Cardin, P. Olson, Geophys. J. Int. 121, 136 (1995)

J. Wicht, Geophys. J. Int. 162, 371 (2005)

J. Wicht, J. Aubert, GWDG-Bericht 68, 49 (2005)

J. Wicht, F. Busse, Geophys. Astrophys. Fluid Dyn. 86, 103 (1997)

J. Wicht, U. Christensen, Geophys. J. Int. (2010, accepted for publication)

J. Wicht, M. Mandea, F. Takahashi et al., Space Sci. Rev. 132, 261 (2007)

J. Wicht, P. Olson, Geochem. Geophys. Geosyst. 5 (2004). doi:10.1029/2003GC000602

J. Wicht, S. Stellmach, H. Harder, in Geomagnetic Field Variations-Space-Time Structure, Processes, and Effects on System Earth. Springer Monograph, ed. by K. Glassmeier, H. Soffel, J. Negendank (Springer, Berlin, 2009), p. 107

A.P. Willis, B. Sreenivasan, D. Gubbins, Phys. Earth Planet. Inter. 165, 83 (2007)

W.-C. Yu, L. Wen, Earth Planet. Sci. Lett. 245, 581 (2006)

S. Zatman, J. Bloxham, Nature 388, 760 (1997)

S. Zatman, J. Bloxham, Geophys. J. Int. 138, 679 (1999)

K. Zhang, G. Schubert, Annu. Rev. Fluid Mech. 32, 409 (2000)

K.-K. Zhang, F. Busse, Geophys. Astrophys. Fluid Dyn. 44, 33 (1988) 